\title{
A review of nonsurgical facial rejuvenation
}

\author{
Stephanie E. Farber ${ }^{1}$, Mathew T. Epps², Emily Brown², Julie Krochonis², Rena McConville², Mark A. \\ Codner $^{2}$ \\ 'Department of Plastic Surgery, University of Pittsburgh, Pittsburgh, PA 15261, USA. \\ ${ }^{2}$ Department of Plastic Surgery, Mark Codner MD Plastic Surgery, Atlanta, GA 30318, USA.
}

Correspondence to: Dr. Stephanie E. Farber, Department of Plastic Surgery, University of Pittsburgh, 3350 Scaife Hall Suite 6B, Pittsburgh, PA 15261, USA. E-mail: stephanie.farber.md@gmail.com

How to cite this article: Farber SE, Epps MT, Brown E, Krochonis J, McConville R, Codner MA. A review of nonsurgical facial
rejuvenation. Plast Aesthet Res 2020:7:72. http://dx.doi.org/10.20517/2347-9264.2020.152

Received: 16 Jul 2020 First Decision: 25 Aug 2020 Revised: 29 Aug 2020 Accepted: 25 Nov 2020 Published: 10 Dec 2020

Academic Editor: James E. Zins Copy Editor: Cai-Hong Wang Production Editor: Jing Yu

\begin{abstract}
As the demand for noninvasive facial rejuvenation continues to grow, it is imperative that plastic surgeons maintain a mastery of nonsurgical techniques for restoring a youthful facial appearance. In this article, noninvasive interventions for skin resurfacing, tissue tightening, rhytid reduction and volume restoration are discussed with an emphasis on technical outcomes and potential complications. Overall, this review should serve as a primer for the aesthetic plastic surgeon who aims to offer safe, effective facial rejuvenation to patients who desire maximal results with minimal downtime.
\end{abstract}

Keywords: Nonsurgical, noninvasive, facial rejuvenation, laser, injectable, radiofrequency skin tightening, ultrasonic skin tightening, skin care

\section{INTRODUCTION}

Over the past decade, plastic surgeons have seen a dramatic increase in the demand for noninvasive rejuvenation procedures. According to American Society of Plastic Surgeons statistics, there were 15.9 million minimally invasive cosmetic procedures performed in 2018 - a 2\% increase from 2017 and a $228 \%$ increase from $2000^{[1]}$. In contrast, there were 1.8 million cosmetic surgical procedures performed in 2018 - a $5 \%$ decrease from 2000. These numbers highlight the fact that the growth of noninvasive procedures outpaces that of surgical procedures. For that reason, it is critical that plastic surgeons are prepared to offer safe and effective nonsurgical rejuvenation to respond to this growing demand. 
Part of the increase in noninvasive procedures can be attributed to the rise in new injectables and technologies offered to patients. Many of the soft tissue fillers, laser treatments, and radiofrequency- or ultrasound-based skin tightening methodologies used today were introduced within the last decade ${ }^{[1]}$. With the growing availability of novel techniques, plastic surgeons are charged with maintaining a masterful knowledge of how to deliver these interventions to optimize outcomes and minimize complications.

This article focuses on the four key age-related facial changes - diminished skin quality, increased soft tissue laxity, formation of rhytids and volume deflation or redistribution - and how each of these can be countered using noninvasive technology - skin resurfacing, soft tissue tightening, rhytid reduction, and volume restoration, respectively. The aim of this review is to arm the aesthetic plastic surgeon with the knowledge and techniques to obtain the best results in the safest, nonsurgical fashion.

\section{SKIN RESURFACING}

\section{Skin care}

\section{Sunscreen}

Sun exposure accelerates intrinsic skin aging by damaging dermal collagen and elastin and interfering with normal skin metabolism. Together, these derangements promote irregular pigmentation, telangiectasias, elastosis, and rhytids ${ }^{[2]}$. However, multiple studies have demonstrated that sunscreen effectively combats further progression of photoaging, while not reversing existing effects ${ }^{[3]}$. Inorganic sunscreens, specifically zinc oxide and titanium dioxide, provide superior, broad-spectrum ultraviolet light protection, increased photostability, and reduced allergenic potential compared to organic sunscreens like aminobenzoates, cinnamates, and salicylates ${ }^{[4]}$. The ideal sunscreen to offer to plastic surgery patients contains the highest percentage of nano- or micro-sized titanium dioxide and zinc oxide to allow for a cosmetically elegant application without the white appearance characteristic of traditional inorganic sunscreens ${ }^{[5]}$. Patients should be instructed to apply sun protection thirty minutes before sun exposure, reapply frequently, and use in conjunction with other sun avoidance habits ${ }^{[6]}$.

\section{Antioxidants}

Antioxidants are intended to protect skin from oxidative stress and to restore skin subjected to environmental damage $^{[7]}$. Vitamin $C$ enhances collagen production and epidermal turnover to improve skin surface appearance, while vitamin E synergistically neutralizes reactive oxygen species ${ }^{[8]}$. The addition of ferulic acid stabilizes these two antioxidants and increases their efficacy in retarding and reversing cutaneous signs of aging ${ }^{[9]}$. The ideal antioxidant should contain a blend of these ingredients and others, such as polyphenols, flavonoids, and carotenoids, in a stable formulation and in a high enough concentration to be effective without causing irritation ${ }^{[4]}$. Patients should be encouraged to use these antioxidants as part of their daily skincare routines.

\section{Retinoids}

Retinoids increase dermal collagen content, epidermal thickness, and glycosaminoglycan deposition. These histologic effects lead to increased skin smoothness and wrinkle reduction ${ }^{[10]}$. The prescription formulation, tretinoin, is limited by compliance issues due to problematic side effects such as redness, irritation, and desquamation that may cause patients to stop treatment if they are not fully educated on how to manage these issues such as with reduction in frequency of application or concentration of medication. However, while these side effects have been shown to be dose dependent, the skin rejuvenating effects are not. Therefore, patients can be prescribed the lowest concentration $(0.025 \%)$ of tretinoin and still expect maximal benefit ${ }^{[10]}$. Retinol, a tretinoin precursor, must be converted to its active counterpart in the skin and is, therefore, 20 -fold less effective and has 1000-fold lower dermal concentration than tretinoin ${ }^{[1]]}$. Retinaldehyde, another tretinoin precursor, has similar efficacy to tretinoin with fewer side effects. However, its long-term benefits have not yet been compared to tretinoin ${ }^{[12]}$. For these reasons, tretinoin 

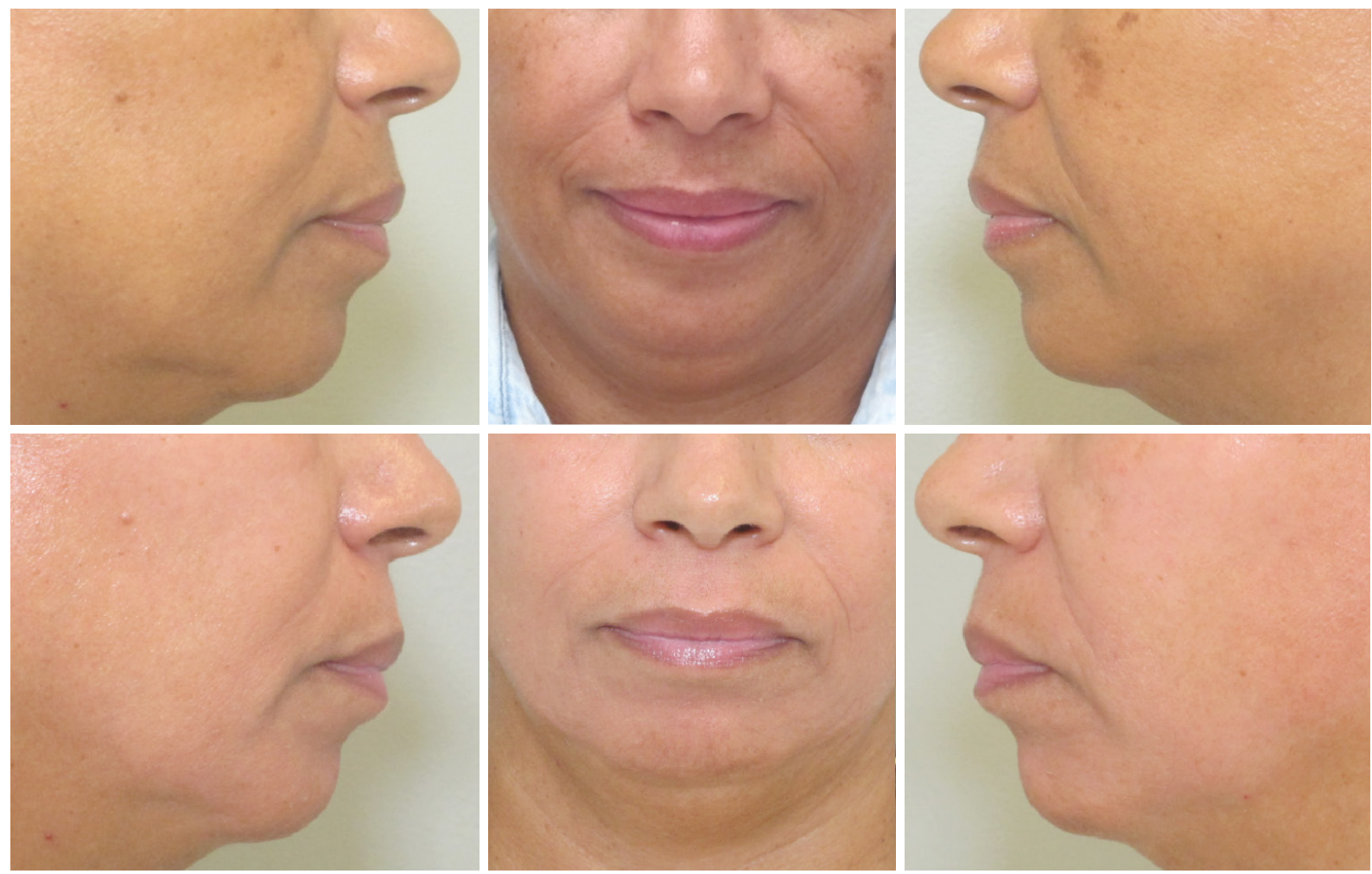

Figure 1. Skincare regimen. 53-year-old Hispanic female treated with a skincare regimen which includes topical retinoid, vitamin C, and hydroquinone. Before (top) and following 5 weeks (bottom) of daily skincare treatment

remains the gold standard retinoid for skin rejuvenation and should be recommended as an adjunct to other aesthetic treatments.

An example of a patient treated with a combination regimen of a retinoid, vitamin $\mathrm{C}$, and hydroquinone is included in Figure 1.

Acids

Alpha hydroxy acids, such as glycolic, lactic, and citric acids, work to exfoliate the skin and interfere with epidermal cohesion, creating a more youthful skin appearance. One study demonstrated an increase in skin thickness with improved elastin quality and collagen density in patients using a combined alpha hydroxy acid formulation ${ }^{[13]}$. Another study showed a dose-dependent improvement in skin smoothness and reduction in rhytids with use of topical lactic acid ${ }^{[14]}$. Patient should be encouraged to use the topical alpha hydroxy acid with the highest effective concentration that they can tolerate without significant redness or desquamation. They should be instructed that acids should not be used with antioxidants as the combination can destabilize vitamin $\mathrm{C}$ and reduce its efficacy ${ }^{[15]}$.

\section{Medical facials}

Dermal infusion facials (such as those offered by HydraFacialÔ, Diamond GlowÔ, and SilkPeel ${ }^{\circ}$ ) include a combination of mechanical exfoliation, suction-based extraction, and active ingredient infusion. The exfoliation technique associated with dermal infusion facials is termed hydradermabrasion and combines microdermabrasion with pressurized application of an ingredient-containing serum. In one study comparing combined hydradermabrasion and antioxidant serum infusion with topical application of the same antioxidant serum, the treatment group was found to have increased epidermal and dermal thickness, increased antioxidant levels, decreased fine lines, decreased pore size, and decreased hyperpigmentation ${ }^{[16]}$. 
Though further study is needed to support these conclusions, dermal infusion facials are a low-risk, appealing adjunct to other nonsurgical rejuvenation techniques.

\section{Chemical peels}

Pretreatment

Chemical peels are a cost-effective means of skin resurfacing that can be tailored to various patients. Prior to treatment, a Fitzpatrick analysis is critical as patients with a Fitzpatrick type of IV through VI are at highest risk of pigmentary abnormalities. These patients should be treated with extreme caution or not at all $^{[17]}$.

Pretreatment increases the effectiveness of chemical peels and should be initiated four to six weeks before treatment. Tretinoin pretreatment results in more uniform frosting and more rapid reepithelialization ${ }^{[18]}$. Hydroquinone should be recommended to patients with Fitzpatrick type III or greater to reduce the risk of post-inflammatory hyperpigmentation ${ }^{[19]}$. Glycolic acid at a concentration of $10 \%$ or less is also administered daily prior to treatment in order to accelerate exfoliation. These are all stopped one week prior to treatment to avoid irritation prior to the peel. Antiviral therapy should also be given to patients with a history of herpes simplex starting two days before and continuing for ten days after ${ }^{[20]}$.

Immediately before treatment, the skin should be de-greased with acetone or rubbing alcohol. Following this, the peeling agent can be applied by facial subunit with gauze or a cotton-tipped applicator and feathered at the edges to blend with untreated areas ${ }^{[21]}$. Application to the eyes, mouth, and alar facial groove should be avoided due to the risk of erosion ${ }^{[22]}$.

\section{Superficial}

Superficial chemical peels are indicated for mild skin texture abnormalities and dyschromias. They have the advantage of minimal to no downtime and are usually used serially to maximize effect. The appropriate superficial peel depth through epidermis only is achieved when a cloudy white frost on a pink background is noted. When this frosting is achieved, the peel should be neutralized if needed. Glycolic acid at a concentration of $30 \%-50 \%$ is superficial peeling agent that must be neutralized with water or a weak base. Jessner solution is another superficial peeling agent that does not require neutralization ${ }^{[23]}$.

\section{Medium}

Medium depth peels are indicated for fine wrinkles and dyschromias. Depth of peel is appropriate when a white frost with erythematous strikethrough is observed, indicating penetration to or through the papillary dermis. At this time, glycolic acid 70\% is neutralized as above and TCA 30\%-50\% does not require neutralization. These peels require between three to seven days of downtime during which patients should expect swelling and erythema ${ }^{[23]}$.

\section{Deep}

Deep chemical peels are indicated for coarse wrinkles and deeper acne scars. Due to their depth of penetration, these peels should be administered with intravenous sedation or regional blocks. Phenol based peels, including Baker-Gordon formula must be performed with cardiac monitoring and intravenous fluids. Depth of peel through the papillary dermis into the reticular dermis is achieved when solid white frosting without background erythema is noted. A grey hue should be avoided as this indicates excessive depth of peeling. Deep chemical peels require ten to fourteen days of downtime and are associated with a more painful recovery ${ }^{[23]}$.

Hetter's solution - the combination of phenol and croton oil to increase penetration - can be performed as a superficial, intermediate or deep peel. The depth is varied by adjusting the number of swipes, amount of solution applying, or concentration of the phenol and croton oil. 
Peel depth will be evident in the nature of the frost, with pink frost indicating an epidermal injury, pinkwhite a papillary dermal injury, and white a reticular dermal injury.

\section{Posttreatment}

Following medium and deep peels, patients are advised to moisturize frequently with petroleum-based cream. Sun avoidance should begin immediately, but application of sunscreen can resume ranging from immediately after a superficial peel to two weeks after a deep peel when re-epithelialization has occurred. Hydroquinone should be initiated by the treating physician immediately at the first sign of hyperpigmentation ${ }^{[23]}$. Milia typically respond to treatment with topical tretinoin ${ }^{[22]}$.

In addition to oral acyclovir in patients with a known history of herpes simplex, patients with prolonged, painful erythema should be treated with two doses of fluconazole out of concern for a yeast infection ${ }^{[24]}$.

\section{Laser}

\section{Resurfacing}

Laser resurfacing induces an epidermal or dermal injury and regeneration, resulting in improved skin tone, effaced wrinkles, and reduced dyspigmentation. Resurfacing should be performed with caution in patients with history of scarring and Fitzpatrick type of $\mathrm{V}$ or greater. The pretreatment regimen is similar to the regimen outlined for chemical peels above, including tretinoin and hydroquinone, as well as acyclovir which may be used selectively for patients with a history of herpes infection or may be used for all patients. Superficial resurfacing can be performed with topical anesthetic, while deeper treatments should be performed under nerve blocks, intravenous sedation, or general anesthesia ${ }^{[25]}$. Resurfacing lasers can be categorized as ablative or non-ablative and fractionated or non-fractionated.

Non-ablative lasers have reduced downtime and lower risk, while ablative lasers have a more dramatic rejuvenating effect ${ }^{[21]}$. Ablative lasers, such as the carbon dioxide $\left(\mathrm{CO}_{2}\right)$ laser and erbium:YAG (Er:YAG) laser, use water as a chromophore and vaporize treated zones, inducing collagen remodeling. $\mathrm{CO}_{2}$ lasers are limited by their prolonged recovery time - occasionally up to six months - and their risk of hypopigmentation. The Er:YAG laser is more specifically absorbed by water-containing tissue and results in less collateral damage. However, because of these characteristics, its skin rejuvenating effect may be less pronounced $^{[21]}$. Non-ablative lasers and non-ablative non-coherent light sources, such as intense pulsed light (IPL), pulsed-dye, and neodymium:YAG (Nd:YAG), generate heat to induce dermal injury and improve rhytids without creating open wounds. The thermal energy is hypothesized to stimulate dermal fibroblasts while keeping the epidermis cool and protecting it from injury ${ }^{[21]}$.

Lasers can also be fractionated or non-fractionated. While fractionated lasers create small columns of injury with unaffected areas between, non-fractionated lasers injure the entire treated area. Fractionated lasers can extend to a deeper level of injury while still having reduced downtime due to the uninjured skin between injured columns. However, for the same reason, multiple treatments between 2-4 weeks apart may be required to achieve the desired result ${ }^{[26]}$.

Of the combinations of laser resurfacing treatments, ablative non-fractionated lasers such as 10,600 $\mathrm{nm}$ $\mathrm{CO}_{2}$ and 2,940 nm Er:YAG have the most dramatic effect with the greatest downtime. On the other end of the spectrum, non-ablative fractionated lasers such as 1,440 nm Nd:YAG are the lowest risk with the least downtime, but also have the most modest effect.

Non-ablative non-fractionated lasers such as 1,319 nm pulsed dye, 1,320 nm Nd:YAG and 1,450 nm diode, as well as ablative fractionated lasers such as $10,600 \mathrm{~nm}$ fractionated $\mathrm{CO}_{2}$ and 2,940 $\mathrm{nm}$ fractionated Er:YAG, serve as a middle ground in terms of effectiveness, safety, and downtime ${ }^{[26]}$. Examples of patients 

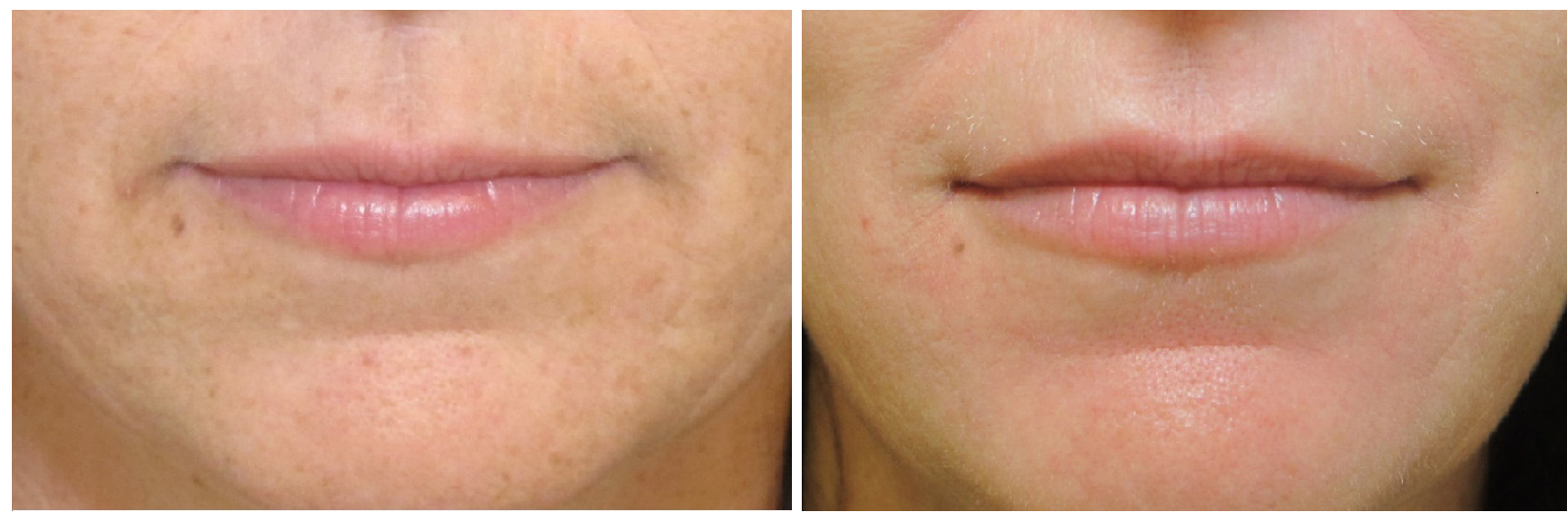

Figure 2. Skincare regimen in combination with pulsed-laser phototherapy [intense pulsed light (IPL)]. 46-year-old Fitzpatrick II Italianportuguese female seeking perioral rejuvenation before (left) and after (right) a combination of daily skincare regimen (topical retinoid, vitamin C, and hydroquinone), hyaluronic acid filler to treat the upper lip rhytids, IPL for three sessions, microneedling to a $2 \mathrm{~mm}$ depth

who underwent pulsed-dye laser resurfacing using IPL, along with a combination of other noninvasive treatments, are shown in Figures 2 and 3. When choosing the correct laser for an individual patient, it is important to consider the patients expectations in terms of desired effect, social downtime, and willingness to undergo multiple procedures.

Posttreatment skincare consists of petrolatum or occlusive dressings until epithelialization is complete, which is between 24-48 h for fractional treatments or a few weeks for full field treatments, at which point a nonocclusive moisturizer can be initiated. Sunblock should also be started as soon as re-epithelialization is complete. Other skin care should be initiated judiciously to prevent irritation ${ }^{[25]}$.

The most common untoward effect of laser resurfacing is erythema, which is an expected part of the healing process. Skin eruptions, such as milia or acne, can occur and usually respond to discontinuation of occlusive agents. Hypopigmentation cannot be treated but can sometimes be camouflaged by treating and reducing pigmentation in other areas. For hyperpigmentation, it can be an expected sequalae of laser treatments in patients with higher Fitzpatrick types or who have early sun exposure, as in Figure 4 In these cases, it is self-limiting. If hyperpigmentation persists, it can be treated with IPL treatments. In rarer situations, burns can occur due to noncompliance with preoperative skincare and sun avoidance, as seen in Figure 5. Other infections rarely occur without the use of prolonged occlusive dressings. If these do occur, they should be treated with the appropriate antiviral, antibacterial or antifungal agents ${ }^{[25]}$.

\section{Other laser indications}

In addition to resurfacing, lasers can also be employed to specifically treat pigmentary and vascular lesions, which are very common sequelae of skin aging. Flat pigmented lesions can be treated using a diode laser at $805 \mathrm{~nm}$ or IPL at $515 \mathrm{~nm}$ with melanin as a chromophore. Lesions with any concern for malignancy should not be treated with laser as this interferes with or prevents pathologic diagnosis. Vascular lesions such as telangiectasias or cutaneous angiomas can be treated with a diode at $805 \mathrm{~nm}$ or IPL at $560 \mathrm{~nm}$ using oxyhemoglobin as a chromophore. These lesions often require multiple treatments ${ }^{[21]}$.

\section{Dermabrasion}

Microdermabrasion

Microdermabrasion is a technique that employs an abrasive component, usually a solid crystalline material, and a vacuum component. Though it is touted as an effective treatment for rhytids, dyspigmentation, and superficial scarring, its effects have been shown to be modest. However, it has been proven to effectively 

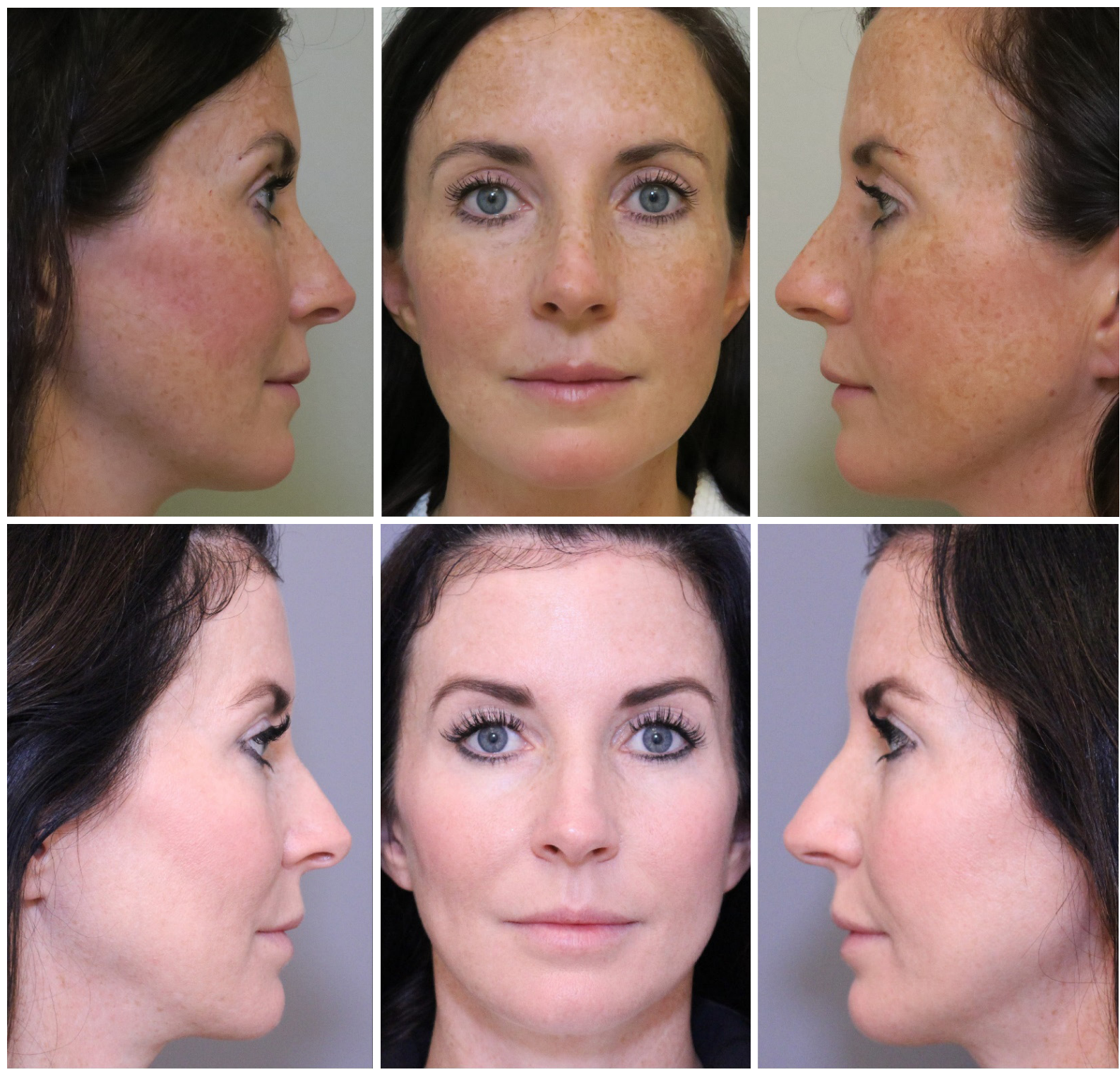

Figure 3. Skincare regimen, pulsed-dye laser resurfacing [intense pulsed light (IPL)], high-intensity focused ultrasound (Ultherapy), and recurrent Botox injections to the glabella, forehead, and crow's feet. 43 yo Caucasian Fitzpatrick II presented with effects of long-term sun damage including dyschromia, rhytids, and mild skin laxity. The patient was initiated on a skincare regimen consisting of topical retinoid, vitamin $\mathrm{C}$, and hydroquinone for four weeks prior to undergoing two sessions of full-face and neck high-intensity focused ultrasound followed by three treatments of pulsed-laser phototherapy (IPL), and recurrent neuromodulator injections to the glabella, forehead, and crow's feet at 3-4 month intervals. Total treatment time was approximately 19 months

increase transdermal delivery of active skincare ingredients when these modalities are combined. The main advantage of microdermabrasion is that, unlike lasers and chemical peels, it is safe in most patients including patients with higher Fitzpatrick types or who are otherwise at higher risk of pigmentary changes or scarring ${ }^{[27]}$.

The procedure begins with facial cleansing and degreasing, followed by three passes in different directions over the treated skin. The degree of dermabrasion depends on the rate of handpiece movement and strength of flow. No anesthetic is required. Following treatment, sunscreen is applied, and there is typically no social downtime. Patients are advised that they will likely undergo multiple treatments. Complications are mostly self-limiting and include erythema, irritation, and petechiae ${ }^{[26]}$. 

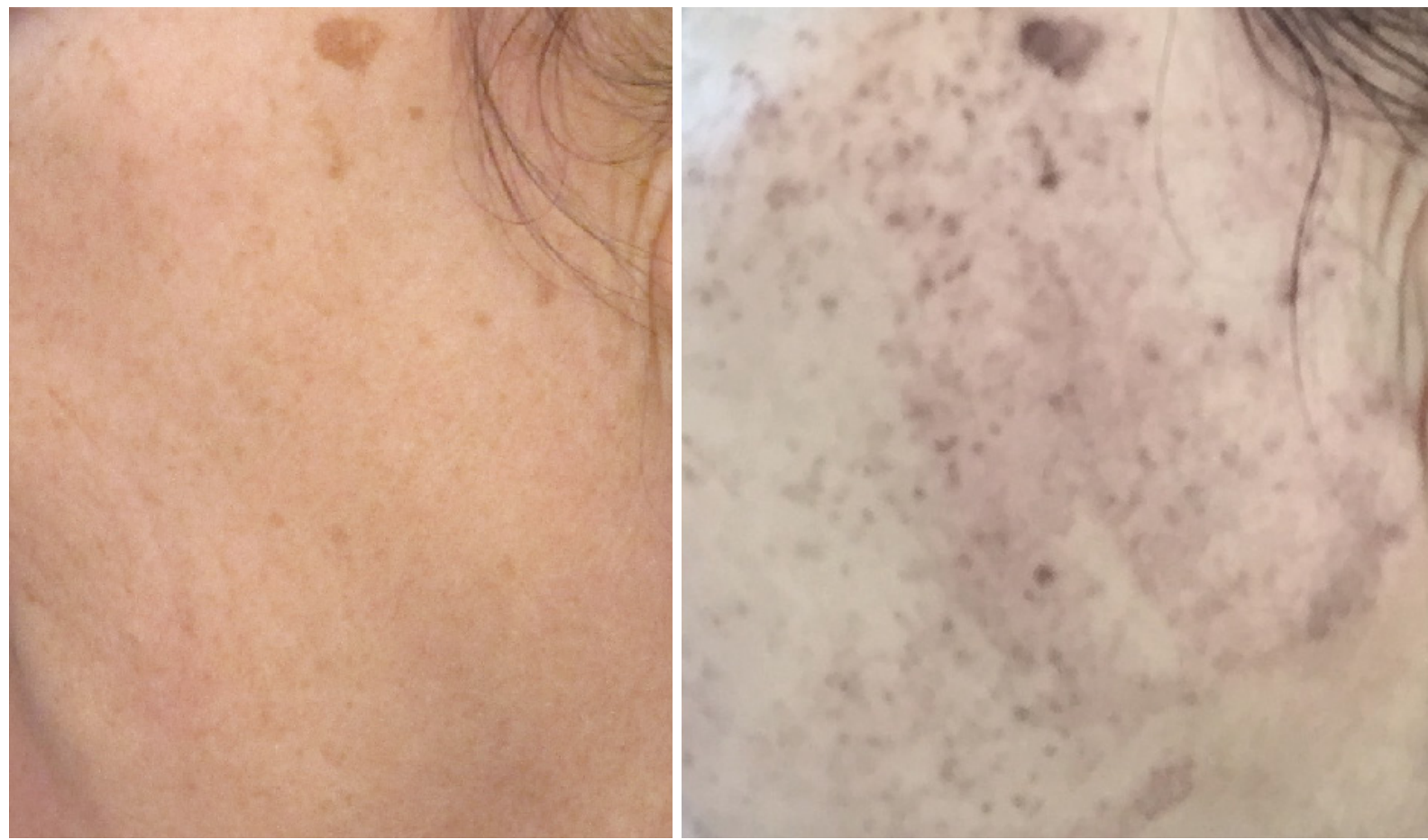

Figure 4. Phototherapy-induced inflammatory hyperpigmentation (PIH). 46-year-old Fitzpatrick II Female pre-treated with our skincare regimen (Tretinoin, Vitamin C, and Hydroquinone 4\%) 1 month prior to intense pulsed light (IPL) phototherapy (left) with a normal physiological hyperpigmentation response to phototherapy (right). PIH normally occurs within $6 \mathrm{~h}$ of IPL treatment and typically resolves at 5-7 days

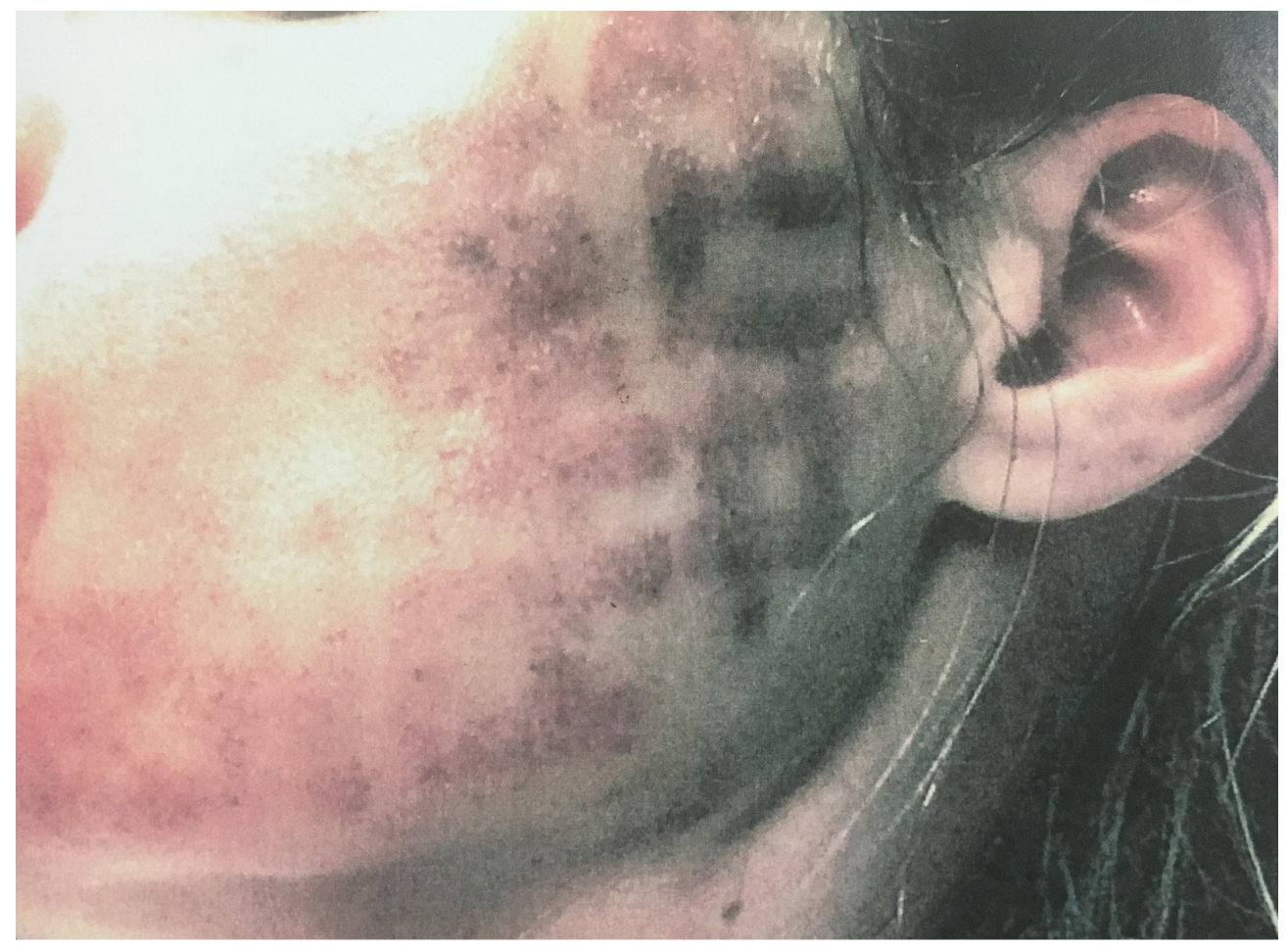

Figure 5. Pulsed-laser phototherapy [intense pulsed light (IPL)] complication. Patient presented for IPL therapy without compliance to preoperative skincare regimen and with extended sunlight exposure over a period of $72 \mathrm{~h}$ prior to IPL therapy. Prior sunlight exposure resulted in a cumulative excess of UV light which resulted in a superficial burn. The burn fully resolved with a short course of topical hydrocortisone cream 


\section{Dermabrasion}

Dermabrasion uses an abrasive motorized wheel to create a mid-dermal wound. It is less frequently used since the advent of laser resurfacing given its increased risk of aerosolized viral particles, dyspigmentation and scarring. It is still an effective option in the treatment of deeper acne scars, but its indications in facial rejuvenation are limited ${ }^{[21]}$.

\section{SOFT TISSUE TIGHTENING}

\section{Radiofrequency}

\section{Mechanism}

Radiofrequency skin tightening works by transmission of thermal energy to the reticular dermis, triggering a remodeling cascade with subsequent collagen, elastin, and vascular formation. It is a non-ablative technology that specifically treats the dermis and spares the epidermis, thereby reducing scarring or pigmentary complications and speeding recovery ${ }^{[28]}$.

\section{Indications and contraindications}

Radiofrequency treatments been shown to be beneficial for the treatment of facial wrinkles, brow lifting, periorbital wrinkles, nasolabial folds, jowls, marionette lines, jawline contouring, and neck laxity ${ }^{[28]}$. Radiofrequency skin tightening is contraindicated in patients with implantable medical devices and those with underlying healing disorders. Multiple studies have shown it to be less effective in older patients, either due to decreased capacity for dermal healing, decreased tissue hydration, or increased severity of preoperative deformity ${ }^{[29]}$.

\section{Procedure}

The administering practitioner must determine whether to treat in one session with higher energy or in multiple sessions with lower energy. One study showed that using lower energy over more sessions was more effective for remodeling of collagen and improvement in skin elasticity ${ }^{[30]}$.

These modalities can be administered through monopolar devices (one electrode with body grounded for unidirectional current flow through the skin such as Thermage ${ }^{\grave{O}}$ ), bipolar devices (two electrodes - one subcutaneous and one percutaneous -with current flowing between such as Body-TiteÔ), and unipolar devices (one electrode without a grounding pad for omnidirectional field of effect administered subcutaneously such as Exilis $\left.{ }^{\grave{ }}\right)^{[31]}$. While monopolar radiofrequency penetrates through all tissue layers and creates deeper tissue injury, bipolar radiofrequency is more localized and offers more superficial treatment, while also being less painful ${ }^{[28]}$. Furthermore, monopolar radiofrequency can be administered under topical anesthetic, while unipolar and bipolar usually require tumescent or general anesthesia ${ }^{[31]}$.

Fractional radiofrequency treatment or radiofrequency microneedling (such as Profound $\mathrm{RF}^{\mathrm{O}}$ and Morpheus $^{\mathrm{o}}$ ) uses microneedle electrodes to deliver fractional radiofrequency energy directly to the reticular dermis, while protecting adnexal structures ${ }^{[28]}$. The ablative effect of the microneedling allows for both a resurfacing and tightening effect and can be beneficial in patients who require treatment of both these signs of aging and are willing to accept a longer recovery than traditional, non-ablative radiofrequency treatments.

\section{Outcomes and complications}

Patients should be instructed that full results of treatment can be expected within six months to one year. The most common adverse events are swelling, numbness, and bruising which generally resolve within 1-2 months and can be treated expectantly ${ }^{[32]}$. 

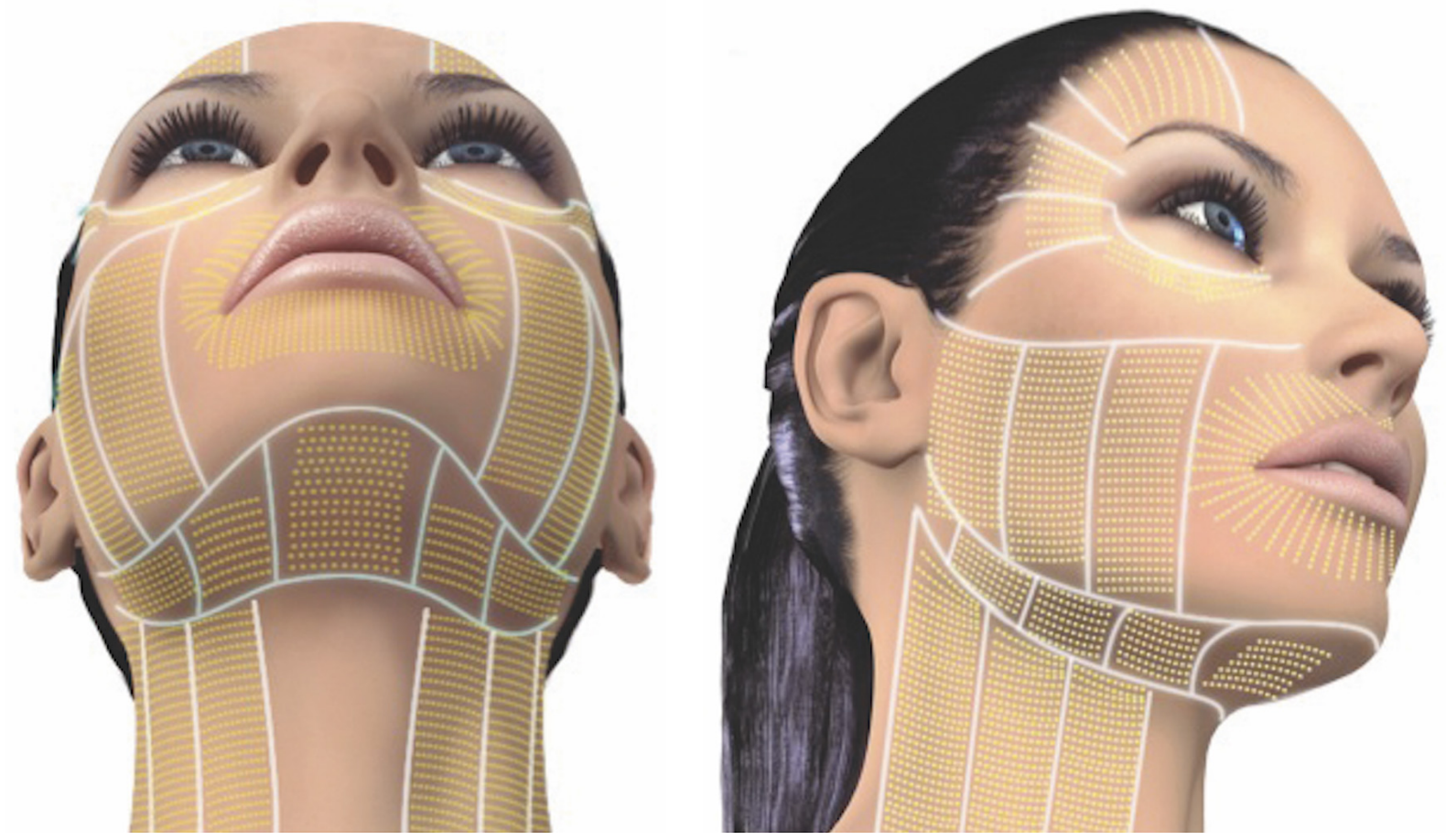

Figure 6. Ultherapy treatment areas. Markings demonstrating the areas and individual yellow ultrasound energy points treated to induce lifting and tightening for the neck, submental region, and cheeks

\section{Ultrasound}

\section{Mechanism}

Ultrasound-based skin tightening (such as Ultherapy $^{\circ}$ ) uses a lower energy, microfocused ultrasound to heat tissue through vibration and friction. This heating produces small coagulation points in the reticular dermis to induce remodeling, while sparing the overlying papillary dermis and epidermis. Like radiofrequency skin tightening, this epidermal sparing reduces the risk and recovery time associated with this procedure. Unlike radiofrequency skin tightening, however, microfocused ultrasound energy can heat deeper tissues without heating the skin, allowing for transmission of higher energies and penetration to the superficial muscular aponeurotic system and platysma ${ }^{[33]}$. It also allows for direct visualization of treated tissues during treatment, adding an additional level of safety ${ }^{[34]}$.

\section{Indications and contraindications}

Ultrasound skin tightening is approved for the eyebrows, chin, neck and chest. The ideal patient has mild to moderate skin laxity and does not have any underlying condition that would inhibit wound healing ${ }^{[34]}$. Because treatment in higher Fitzpatrick types has not been thoroughly studied and because two cases of hypopigmentation have been reported, ultrasound based skin tightening should be used with caution in patients with Fitzpatrick type V or $\mathrm{VI}^{[35]}$. Like radiofrequency skin tightening, microfocused ultrasound has been shown to be less effective in older patients with more severe age-related deformities ${ }^{[33]}$.

\section{Procedure}

The procedure is performed following application of topical anesthetic. Given the moderate to severe pain associated with this treatment, some options for additional anesthetic include nerve blocks, nitrous oxide gas, and oral anxiolytics. Focal depth is visualized on the monitor and calibrated to correspond to the layer intended to be treated before delivering a linear array of ultrasound pulses. Most therapy platforms have pre-programmed protocols for specific treatment areas, as shown in Figure 6, and treatment times vary from 30-60 min per region ${ }^{[34]}$. 

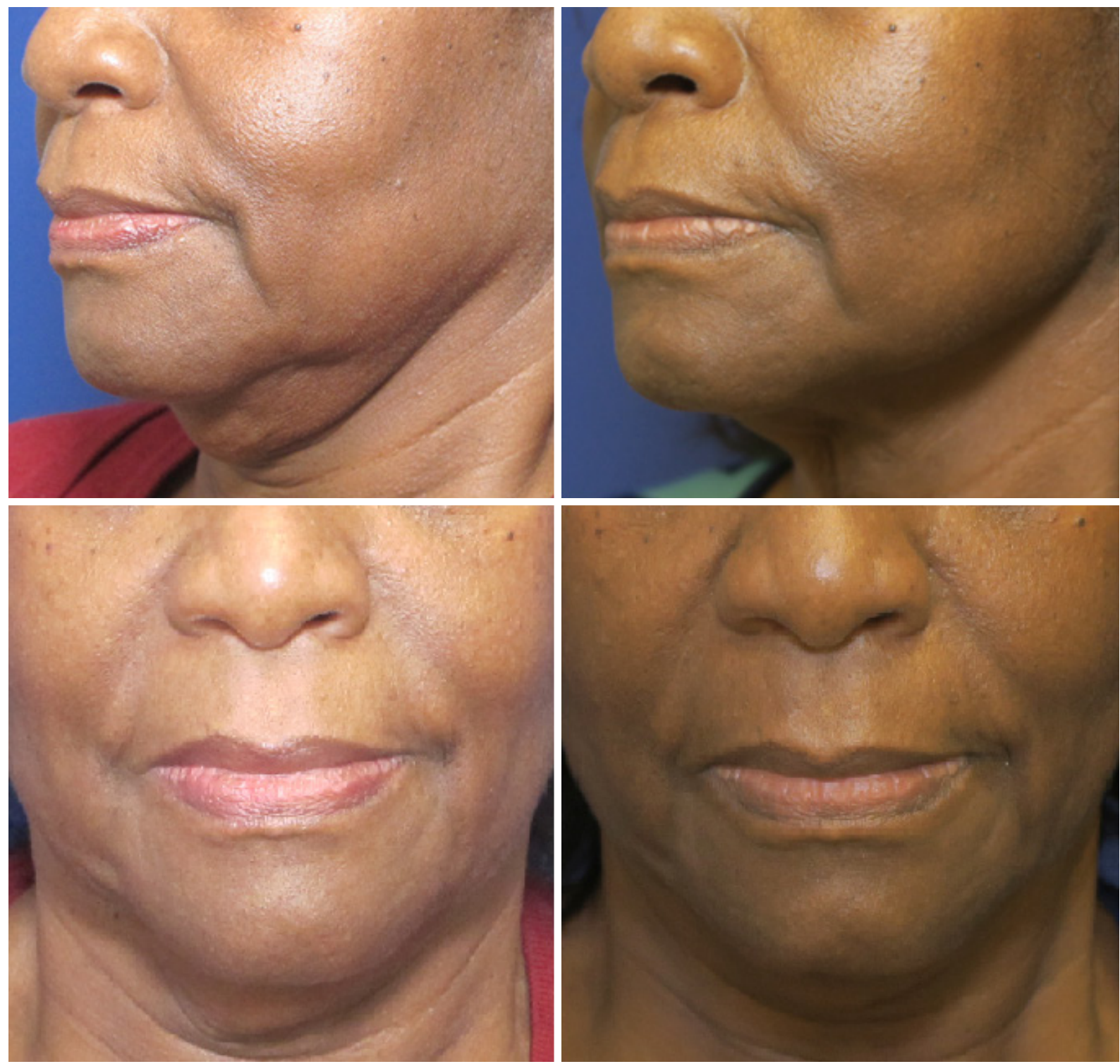

Figure 7. High-intensity focused ultrasound (Ultherapy). 64-year-old African American female before (left) and 2 months following (right) High-intensity focused ultrasound therapy to the neck, cheeks

\section{Outcomes and complications}

Following treatment, patients should expect swelling, erythema, and transient bruising, all of which are self-limiting. Complete results can usually be expected within six months ${ }^{[33]}$. Studies report that greater than $80 \%$ of patients and providers note an improvement following treatment ${ }^{[34]}$. An example of a patient treated with high-intensity focused ultrasound is included in Figure 7. Other examples of patients who underwent a combination of ultrasonic skin tightening and skin resurfacing is shown in Figures 8 and 9.

Rarely, patients can develop linear patterns of skin discoloration that resolve within a few weeks. There have been reports of motor nerve paresis, specifically in the temporal and marginal mandibular branches of the facial nerve, but these have all resolved within six weeks ${ }^{[36]}$. 

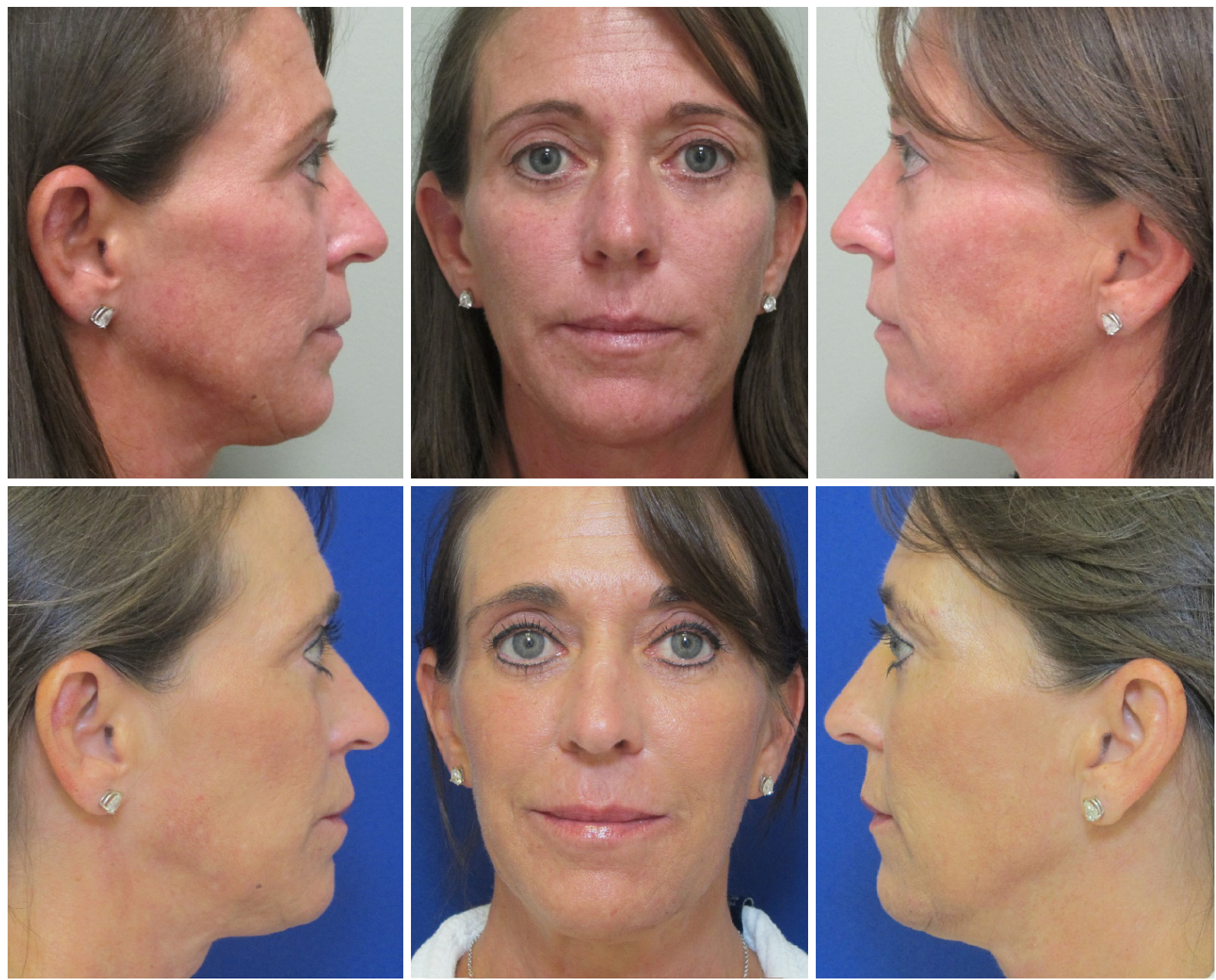

Figure 8. Fractionated bipolar radiofrequency sublative skin resurfacing (eMatrix) and high-intensity focused ultrasound (Ultherapy). 56 yo Caucasian Fitzpatrick III with a history of lower lid blepharoplasty and full-face TCA peel presented with fine facial rhytids and mild facial ptosis. Before (top) and after (bottom) full face fractionated bipolar radiofrequency skin resurfacing (eMatrix) and high-intensity focused ultrasound (Ultherapy) to the neck, face, and brow

\section{Infrared light energy}

\section{Mechanism}

Of the noninvasive skin tightening modalities, infrared technology (such as SkinTyteÔ) is the least well studied. The procedure works by delivering infrared energy via broadband light to create heat in the dermis and initiate collagen remodeling.

\section{Indications}

Unlike ultrasound-based skin tightening, infrared energy does not penetrate to the deeper subcutaneous tissues. Therefore, it is indicated only in mild forms of skin laxity that do not involve the underlying tissue. Similar to other modalities, it should be used with caution in patients with higher Fitzpatrick types.

\section{Procedure}

Typically, infrared skin tightening is not painful enough to require any form of anesthetic. It is performed by heating individual treatment zones to a particular temperature by performing a series of treatment cycles and skin temperature measurements. The skin is then allowed to cool or cool compresses are applied ${ }^{[37]}$. 

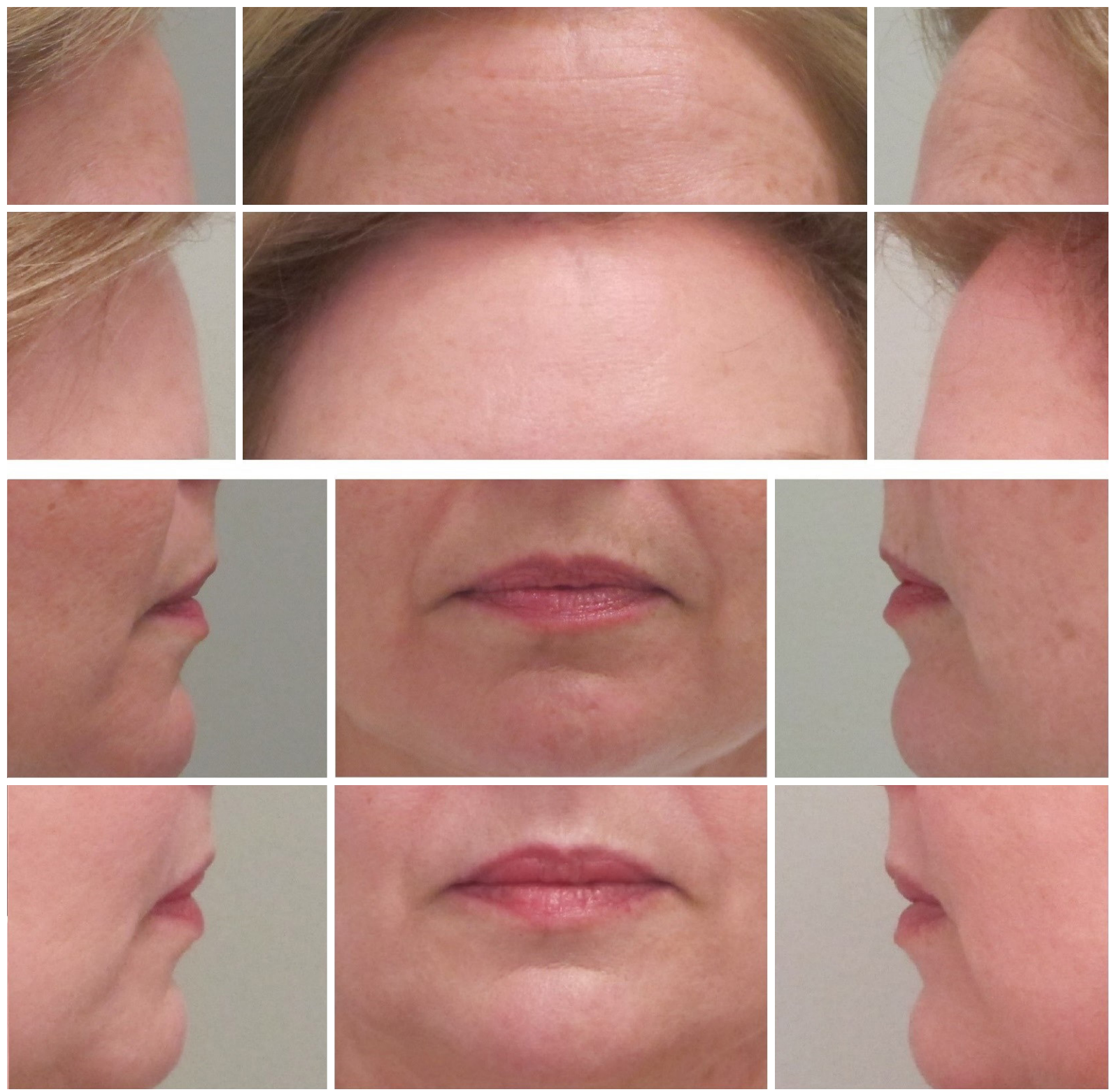

Figure 9. Skincare regimen, fractionated bipolar radiofrequency sublative skin resurfacing (eMatrix) and high-intensity focused ultrasound (Ultherapy). 48 yo caucasian Fitzpartrick II, before (upper top, lower top) and after (upper bottom, lower bottom), a series of three full-face fractionated bipolar radiofrequency sublative skin resurfacing treatments, a single full-face high-intensity focused ultrasound treatment, $1 \mathrm{~mL}$ Hyaluronic acid filler to the nasolabial folds, and recurrent injections of neuromodulator to the glabella, forehead, and crow's feet. Marked improvement in skin texture, dyschromia, and fine rhytids are seen. Treatments occurred for a period of 12 -months

\section{Outcomes and complications}

Usually patients experience mild discomfort post-treatment. Full results should be expected after three to six treatments performed at two- to four-week intervals. Results are variable and outcome and complication data is not well published ${ }^{[37]}$.

\section{Microneedling}

\section{Mechanism}

Microneedling (such as with Skinpen ${ }^{\circ}$ and Dermapen $^{\circ}$ ) is performed by breaching the epidermis with micron-sized needles to create an injury to the outermost layer of the skin. These micro-punctures are touted as a means of injuring the skin and causing elastin and collagen production and deposition ${ }^{[38]}$. 

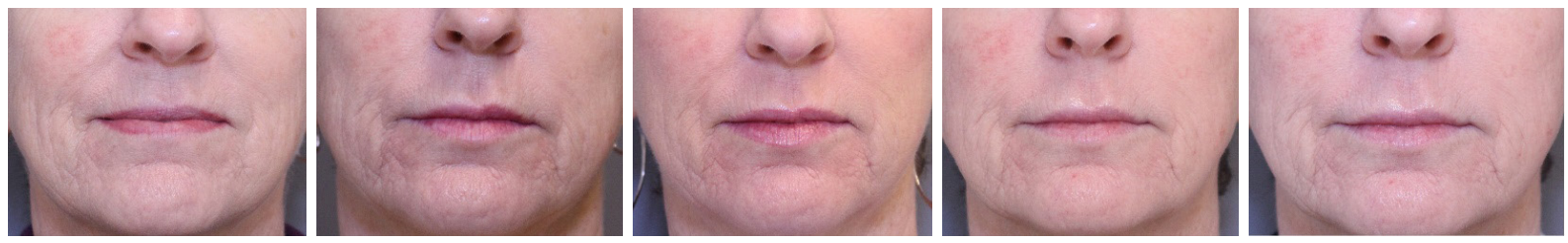

Figure 10. Skincare regimen and microneedling (x3). 61 yo Caucasian female Fitzpatrick I skin before (left) and after (second from left) skincare regimen (topical retinoid, vitamin C, hydroquinone) with $1 \mathrm{~mL}$ hyaluronic acid filler to the lips, followed by microneedling procedures. Shown at one-week post-needling (middle), two-week post-needling (second from right), and three-week post-needling (right). Total course of treatment was approximately 12 months

\section{Indications}

Because the treatment is so superficial, it has only been shown to treat very superficial rhytids ${ }^{[39]}$. A newer application of microneedling is for drug delivery, which will be discussed in a later section.

\section{Procedure}

The procedure is performed by cleansing and applying a topical anesthetic. The microneedling device is applied to the skin in a perpendicular fashion and the device is moved in multiple directions to evenly distribute passes. Uniform, pinpoint bleeding is the endpoint of the procedure, at which point in time a moisturizing sunblock is applied routinely for at least forty-eight hours or indefinitely ${ }^{[40]}$.

\section{Outcomes and complications}

Treatments can be repeated every two to four weeks until desired effect is achieved ${ }^{[40]}$. As seen in Figure 10, effects are modest secondary to the superficial level of injury. However, this superficial penetration also reduces the downtime and risk profile associated with this treatment, with the primary adverse effects being bruising, erythema, and irritation that are self-limiting ${ }^{[41]}$.

\section{RHYTID RELAXATION OR REDUCTION}

\section{Neuromodulators}

Botulinum toxin is the most commonly used nonsurgical facial rejuvenation modality in the United States $^{[1]}$. The toxin works to temporarily paralyze facial muscles, thereby eliminating dynamic rhytids and reducing static ones. Multiple formulations of the toxin are commercially available currently (e.g., Botox ${ }^{\mathrm{O}}$, Dysport $^{\mathrm{O}}$, Xeomin ${ }^{\mathrm{O}}$, Jeaveau ${ }^{\circ}$ ), with additional preparations set to be released (e.g., Daxi). The principle difference in these products is their content of proteins that may cause sensitivity or resistance over time. A meta-analysis of studies comparing three formulations of botulinum toxin failed to find any significant difference in treatment response but concluded that further comparison study is needed ${ }^{[42]}$.

Botulinum toxin treatment is generally safe, but is contraindicated in patients who have allergies to eggs or albumin, who have an infection at the injection site, and who are pregnant or breastfeeding. Relative contraindications include use of anticoagulation given the presumed elevated bleeding risk, underlying neuromuscular disorder given the possibility of increasing severity, or blepharoptosis given the risk of worsening with frontalis paralysis ${ }^{[43]}$.

A general schematic of botulinum toxin injection sites is included in Figure 11 with additional detail below $^{[44,45]}$.

\section{Upper face}

Forehead and periorbital botulinum toxin injections should be performed with careful attention to brow morphology, as each injection will have a resulting impact on brow position. An illustration of how each muscle impacts brow position is show in Figure 12. 


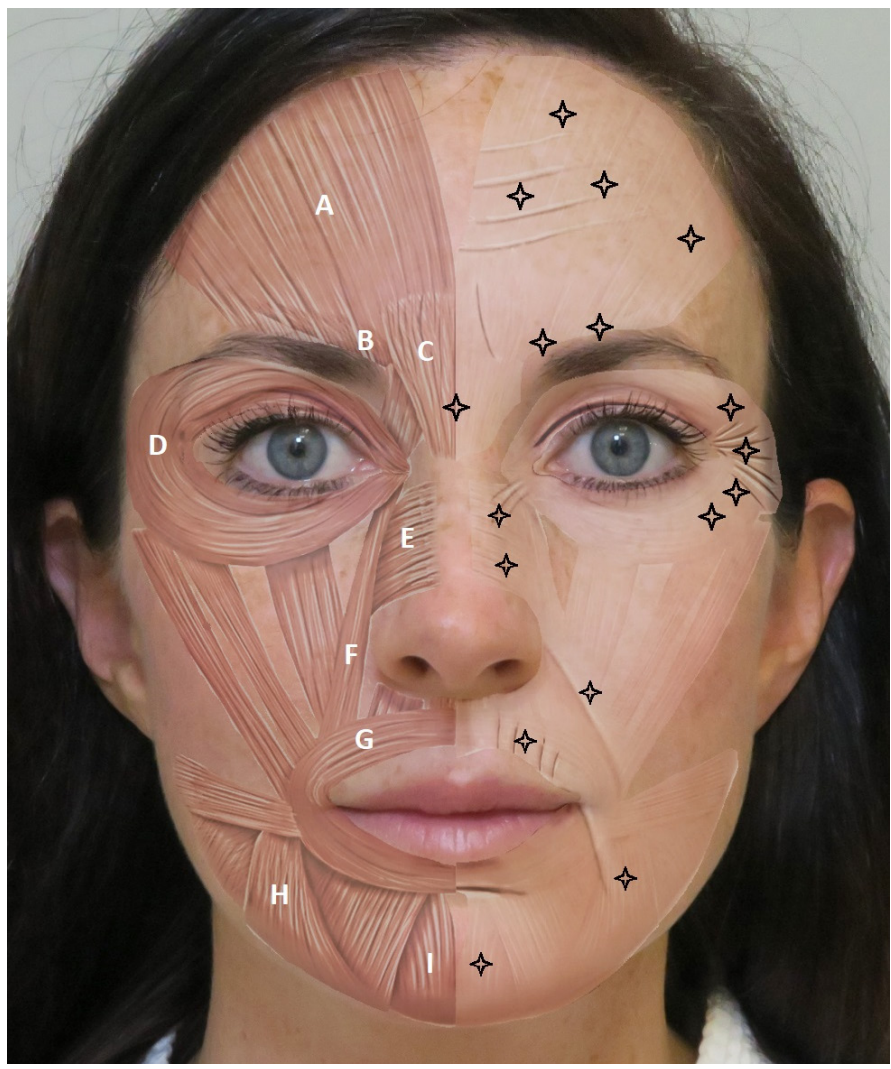

\begin{tabular}{|c|c|c|}
\hline Muscle/muscle group & Muscle action & Static/dynamic rhytids \\
\hline Frontalis (A) & Elevation brows & Horizontal forehead lines \\
\hline $\begin{array}{l}\text { Glabellar muscles: } \\
\text { Corrugator supercilii (B) } \\
\text { Procerus (C) }\end{array}$ & $\begin{array}{l}\text { Medial Elevation of brows } \\
\text { Depressor of brows }\end{array}$ & Frown lines, glabellar creases \\
\hline Orbicularis Oculi (D) & $\begin{array}{l}\text { Elevation of eyelids, concentric tightening of } \\
\text { periorbital tissue }\end{array}$ & Crow's feet \\
\hline Nasalis (E) & Medial elevation of nasal skin & Bunny lines \\
\hline Levator labii superioris alaeque nasi (F) & Elevation of the central lip & Gummy smile, nasolabial Folds \\
\hline Orbicularis oris $(G)$ & $\begin{array}{l}\text { Lip pursing, lower lip and corner of mouth } \\
\text { depressor }\end{array}$ & Radial lip lines, smoker's lines \\
\hline Depressor anguli oris $(\mathrm{H})$ & Corner of mouth depressor & Down turned smile, marionette lines \\
\hline Mentalis (I) & Elevation of chin and lower lip & Dimpled chin, Chin lines \\
\hline Common Neuromodulator Injection Sites & & \\
\hline
\end{tabular}

Figure 11. Muscles of facial expression, their actions, corresponding wrinkles, and points of neuromodulator injection ${ }^{[4]]}$

The frontalis, which creates transverse forehead rhytids, as seen in Figure 13, should be injected in 1.25-2 unit aliquots, depending on the injector's preferred dilution. Injections should be deep given the anatomy of the frontalis muscle and should be planned based on the individual patient's forehead rhytid pattern. To maintain an aesthetic brow shape, injections should not extend too inferiorly or laterally to preserve brow height and arch.

The glabellar muscles, which create mid-brow rhytids, as seen in Figure 14, include the corrugator and procerus. These can be injected in a V pattern with 4-5 units of botulinum toxin per injection. The plane of injection should be deep in order to preserve frontalis in this area. The specific injection pattern should be titrated to the individual patient's rhytid morphology when animating. 


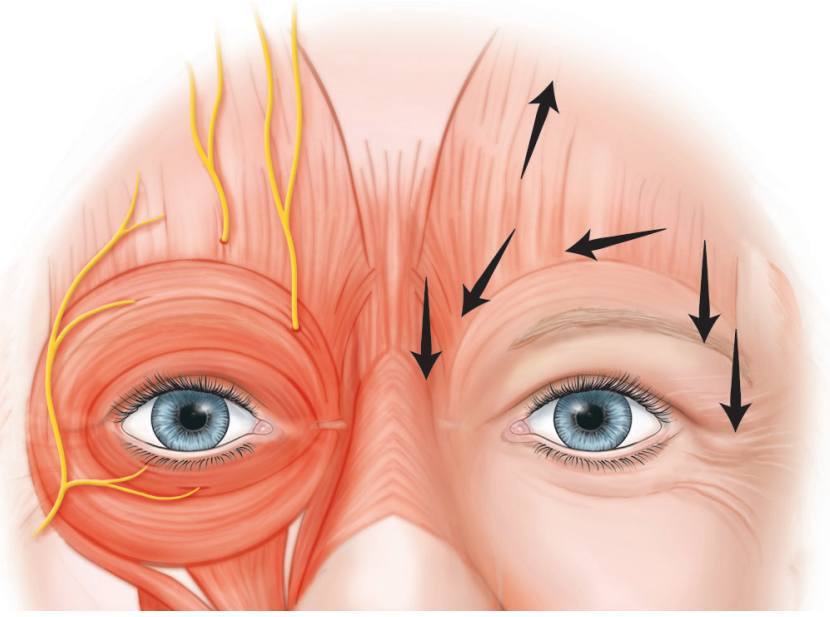

Figure 12. Upper face muscle pull on eyebrow. An illustration of the direction that each upper facial muscle pulls on the eyebrow, showing the medial glabellar muscles exerting a downward force, the frontalis exerting an upward force, and the orbicularis oculi exerting a downward force
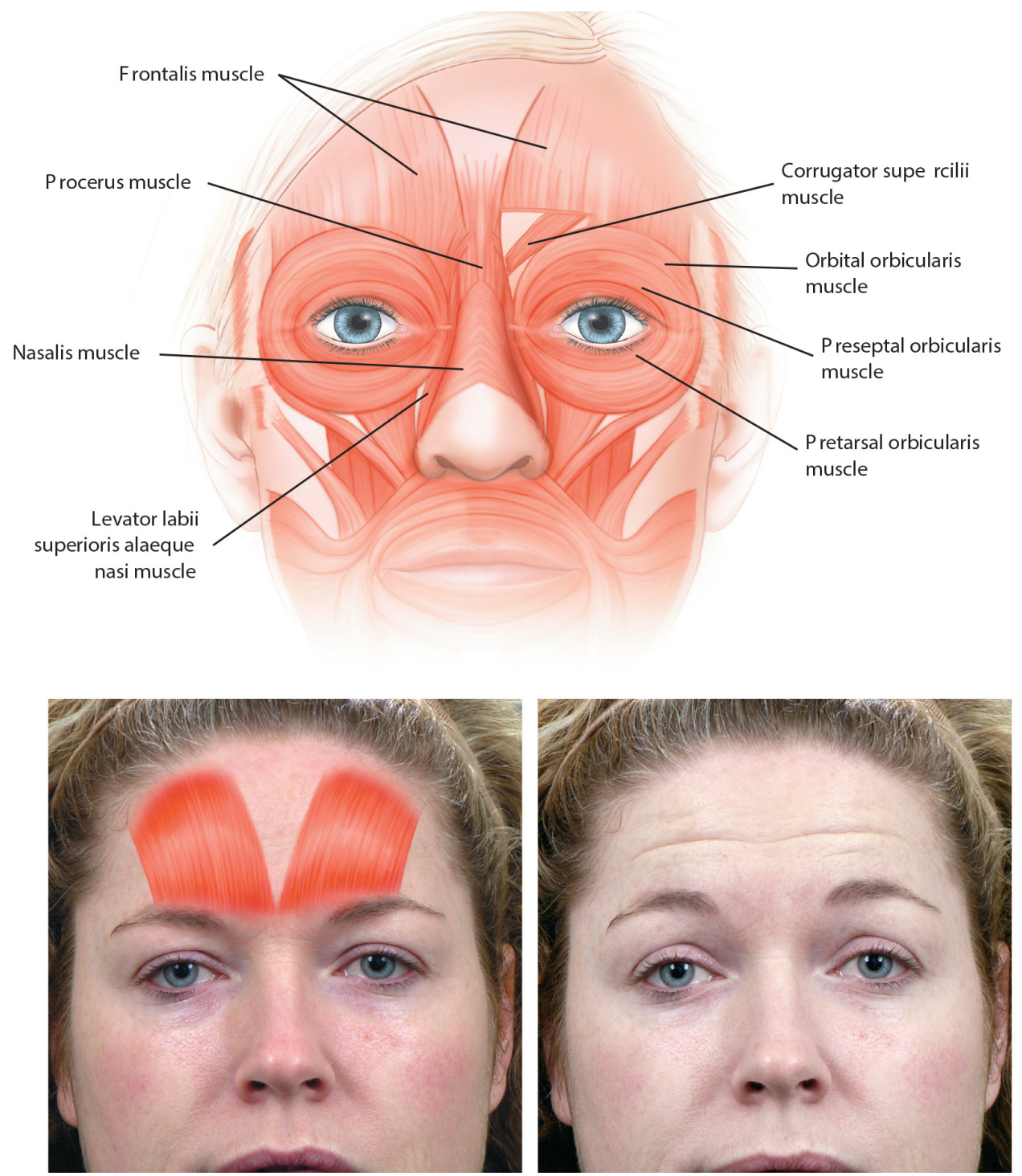

Figure 13. Frontalis muscle anatomy. Facial muscle anatomy (top) and morphology, as seen with animation (bottom) 

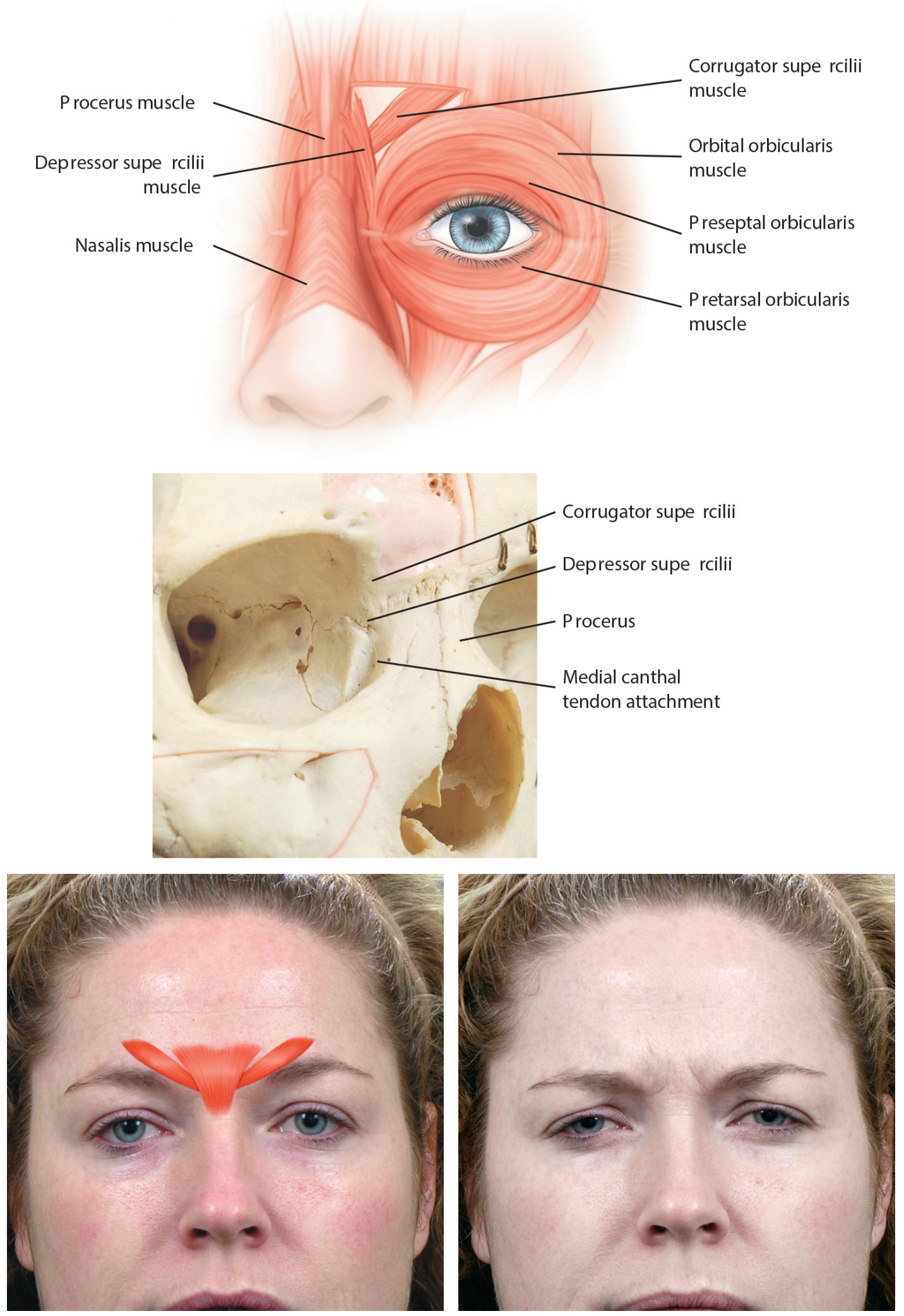

Figure 14. Glabellar muscle anatomy. The corrugator and procerus are seen anatomically (top) with their bony attachments illustrated (middle) and their functional rhytid pattern (bottom) 

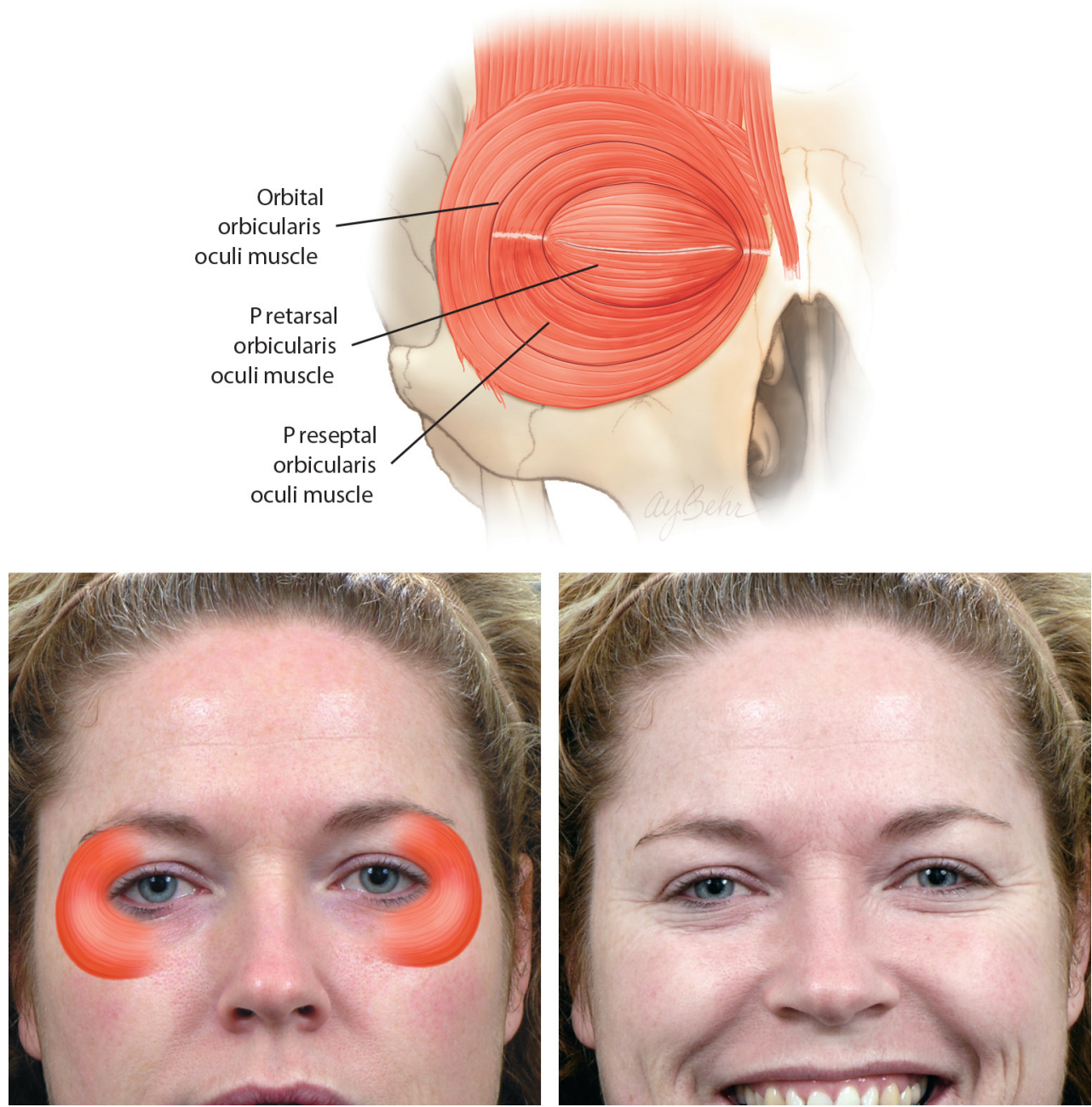

Figure 15. Orbicularis oculis anatomy. Anatomic drawing of the orbicularis oculi muscle (top) and functional anatomy showing dynamic rhytids (bottom)

The orbicularis oculi, Figure 15, should be injected very superficially to target the muscle and avoid spread to the levator palpebrae superioris. These injections serve to obliterate lateral orbital rhytides and to raise the lateral brow. Injections should be performed in 1-2 unit aliquots in three to five sites. Patients with very active muscles may require a second row of injections ${ }^{[46]}$.

Complications of upper facial botulinum toxin injections are undesirable but reversible based on the temporary effect of treatment. Brow asymmetries or overly peaked brows can be managed with additional, judicious injections. Blepharoptosis, however, is not reversible for the duration of treatment effect and can only be mitigated with alpha-adrenergic activation of Muller's muscle to temporarily elevate the eyelid (e.g., with apraclonidine drops). Other adverse effects such as swelling, bruising, or redness are self-limiting ${ }^{[43]}$.

\section{Lower face and neck}

Botulinum toxin injection in the lower face and neck is more difficult and less reliable than in the upper face. Therefore, knowledge of anatomy is critical for using this treatment safely and effectively.

Treatment of the orbicularis oris softens fine vertical perioral rhytids that occur with animation. It is performed by injecting very superficially in two points on the superior and inferior lip at the vermilion 
border. Ideally, a small amount and volume of product should be injected to minimize diffusion (e.g., less than $0.1 \mathrm{~mL}$ of concentrated toxin). It will not treat the static rhytids that occur due to chronological aging or smoking as these are better managed with resurfacing treatments.

Injection into the depressor anguli oris raises the oral commissures. Injections should be performed a few millimeters above the mandibular border just anterior to the jowl at two sites per side, each with 2-2.5 units.

The mentalis muscle can be addressed to reduce its cobblestoning effect on the chin. It is injected at one point per side, five millimeters lateral to midline and one centimeter above the tip of the chin using 2-2.5 units per injection.

Injection of the platysma muscle softens platymsal bands. Usually several (up to ten) superficial injections of 2-2.5 units are required in a vertical line on each side at the anterior border of the muscle with one to two centimeters between injection sites. This technique is facilitated by pinching the muscle between the injector's fingers. Treatment of this area can also help to elevate the oral commissures.

Adverse effects of botulinum toxin in the lower face can be debilitating and are difficult to treat. These can include smile asymmetries, speaking difficulties, drooling, and dysphagia. Safe injection technique and mastery of the relevant anatomy will help to reduce the risk of complications ${ }^{[47]}$.

\section{VOLUME RESTORATION OR REDISTRIBUTION}

\section{Restoration}

Fillers

Injectable soft tissue fillers can be either resorbable (hyaluronic acid and poly-L-lactic acid) or nonresorbable (calcium hydroxyapatite and polymethylmethacrylate). Due to its enzymatic reversibility in the face of adverse outcomes, hyaluronic acid is the most commonly used filler. Its degree of cross-linking determines its viscosity and indications. Specifically, the more highly cross-linked formulations are more viscous and are indicated for deeper injections, whereas the less cross-linked formulations are thinner and indicated for more superficial injections ${ }^{[48]}$. A schematic of the layer of injection for higher and lower degrees of cross-linking is shown in Figure 16.

In the upper face, filler can be used to elevate the lateral brow by injecting into the deep tissue of the lateral eyebrow at the inferior aspect of the supraorbital rim using $0.25-0.5 \mathrm{~mL}$ of product [Figure 17]. It can also be injected into the upper lid sulcus deep to the orbicularis to correct an A-frame deformity ${ }^{[49]}$. The tear trough is more difficult to correct due to the thin skin and high risk of discoloration or contour irregularities. The safest method to treat this area is to place a $0.25 \mathrm{~mL}$ aliquot of a low cross-linked filler along the preperiosteal infraorbital rim rather than injecting directly into the tear trough. Various injection strategies for correction of the tear trough deformity are shown in Figure 18. All areas are massaged following injection to create a smooth, even contour ${ }^{[48]}$.

In the midface, cheek augmentation can be achieved by performing small volume injections along the zygoma at the level of the periosteum beginning lateral to the midpupillary line with subsequent massage to smooth the filler. In this area, a more highly-crosslinked filler should be used given the depth of injection. Nasolabial folds are treated by injecting the mid to deep dermis with a medium cross-linked filler ${ }^{[49]}$ [Figure 19].

In the lower face, the lips can be injected to create a more full, everted youthful appearance. Many patients require small aliquots of local anesthesia or nerve blocks for this procedure. Lip augmentation can be performed using many techniques, and the best is one that is tailored to the patient's unique anatomy and asymmetries. A lower viscosity filler should be used and aesthetic lip proportions should be respected, 


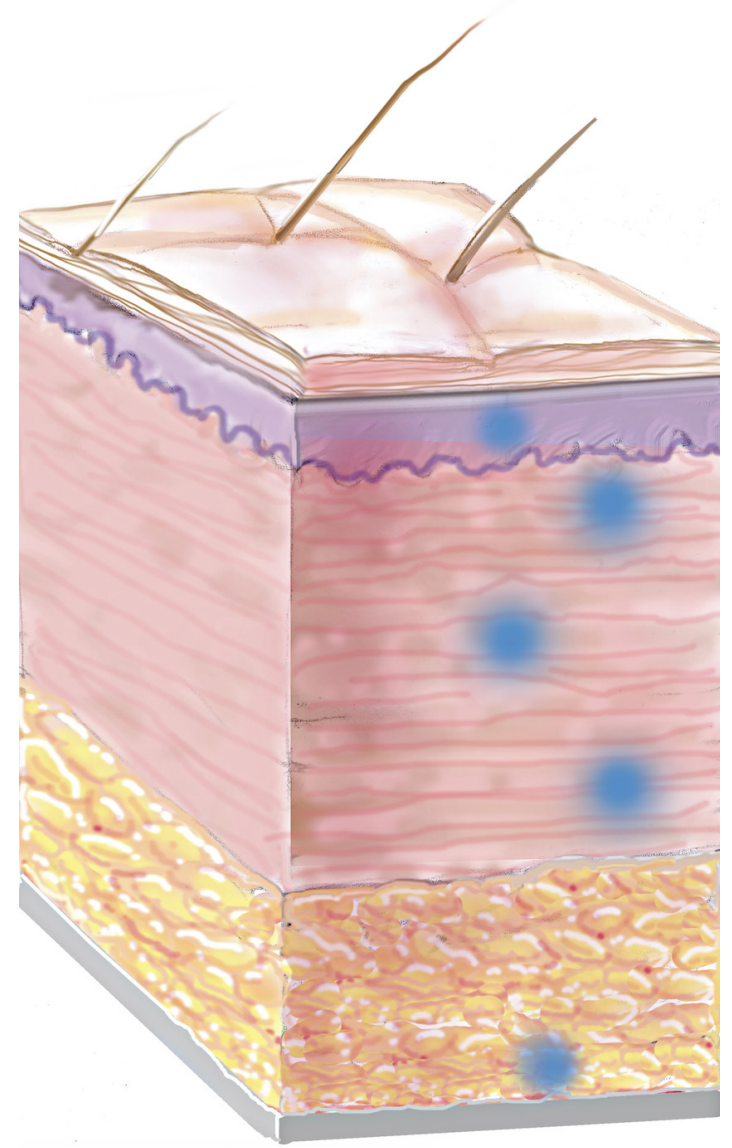

Figure 16. Filler injection depths. Blue areas illustrate depths of filler injection from intradermal (top), to various levels of intramuscular (middle three), and preperiosteal (bottom)

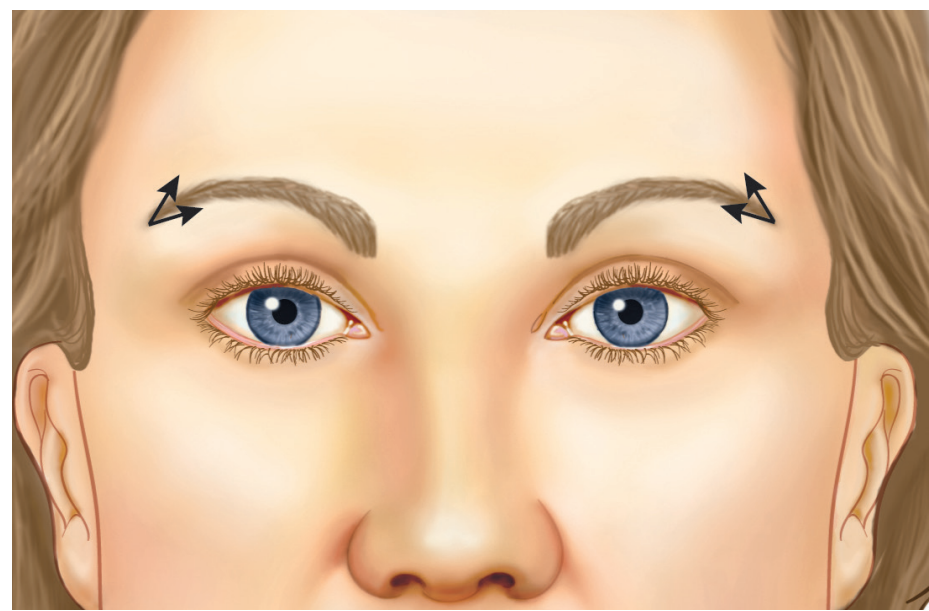

Figure 17. Filler for lateral brow elevation. Location and direction of lateral brow filler injections for brow elevation

injecting along the white roll, within the vermilion and below the commissure. Some patients also benefit from philtral injections. Marionette lines and the pre-jowl sulcus can be treated by a superficial subcutaneous injection of a medium cross-linked filler material. Finally, a recessed chin can be augmented using a supraperiosteal bolus of a highly cross-linked filler material at three sites across the lower chin $^{[50]}$. 

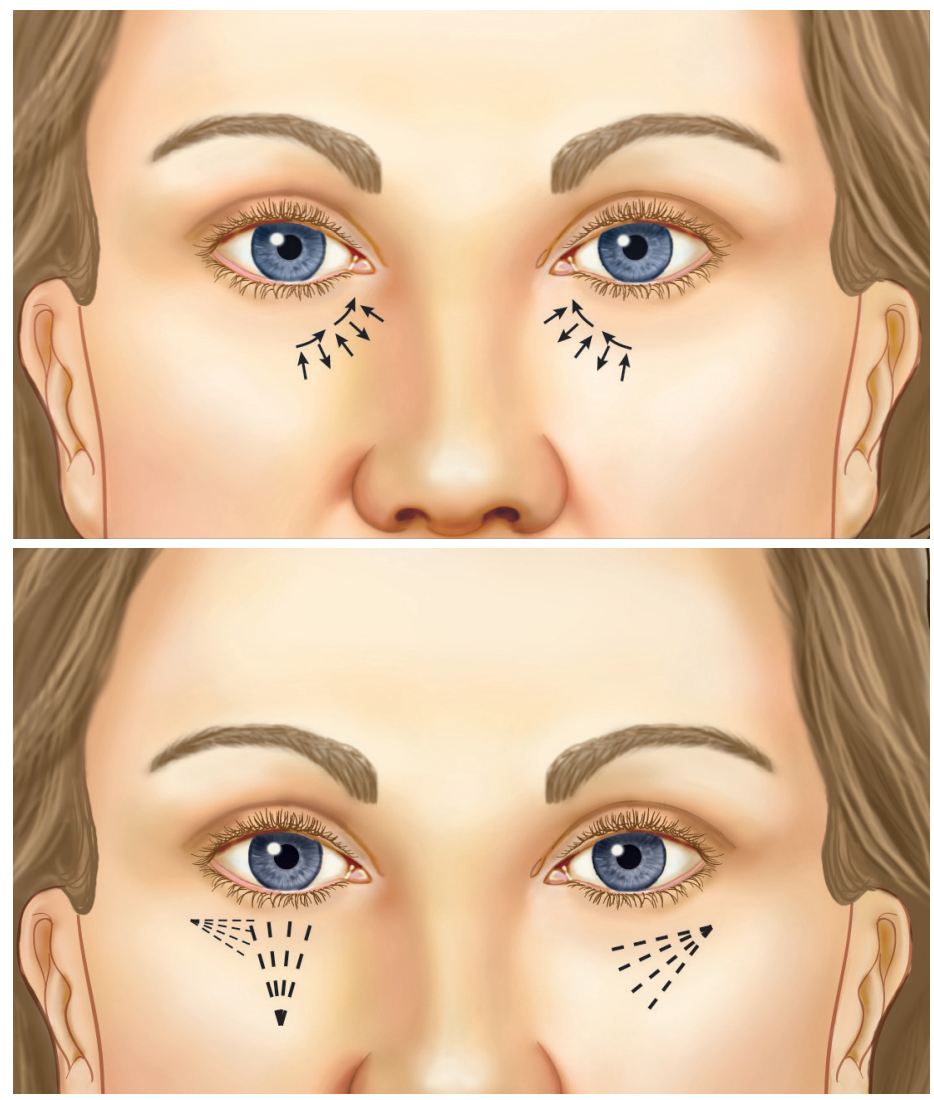

Figure 18. Tear trough filler injection strategies. Various strategies for using filler to correct the tear trough deformity are shown including injection along the tear trough and along the infraorbital rim (top) and within the volume defect in the midcheek (bottom)

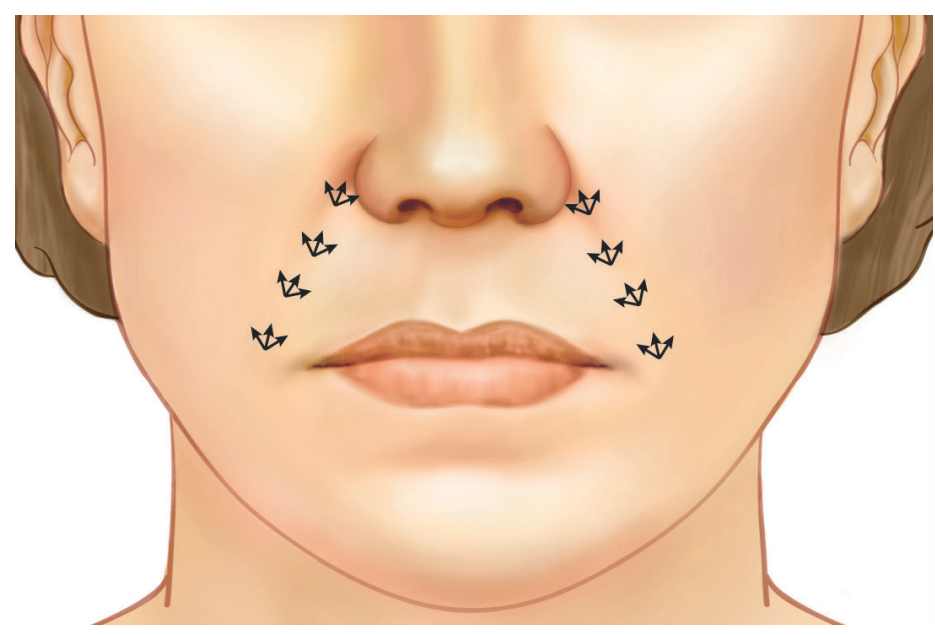

Figure 19. Nasolabial fold filler injection. The directions and pattern of injection for correcting nasolabial fold

Mild adverse events following filler injection include bruising, edema, and erythema that are self-limiting. A herpes flare is a more concerning reaction, which highlights the importance of thorough preoperative history taking to determine the need for pre- and postprocedural prophylaxis. Nodules may form if too much filler is injected in one area or if highly cross-linked fillers are placed too superficially. A trial of massage can be initiated followed by dissolution of the nodule with hyaluronidase (20 units of enzyme for every $0.1 \mathrm{~mL}$ of filler). Most of the dissolution should occur within a few minutes of injection. Vascular 


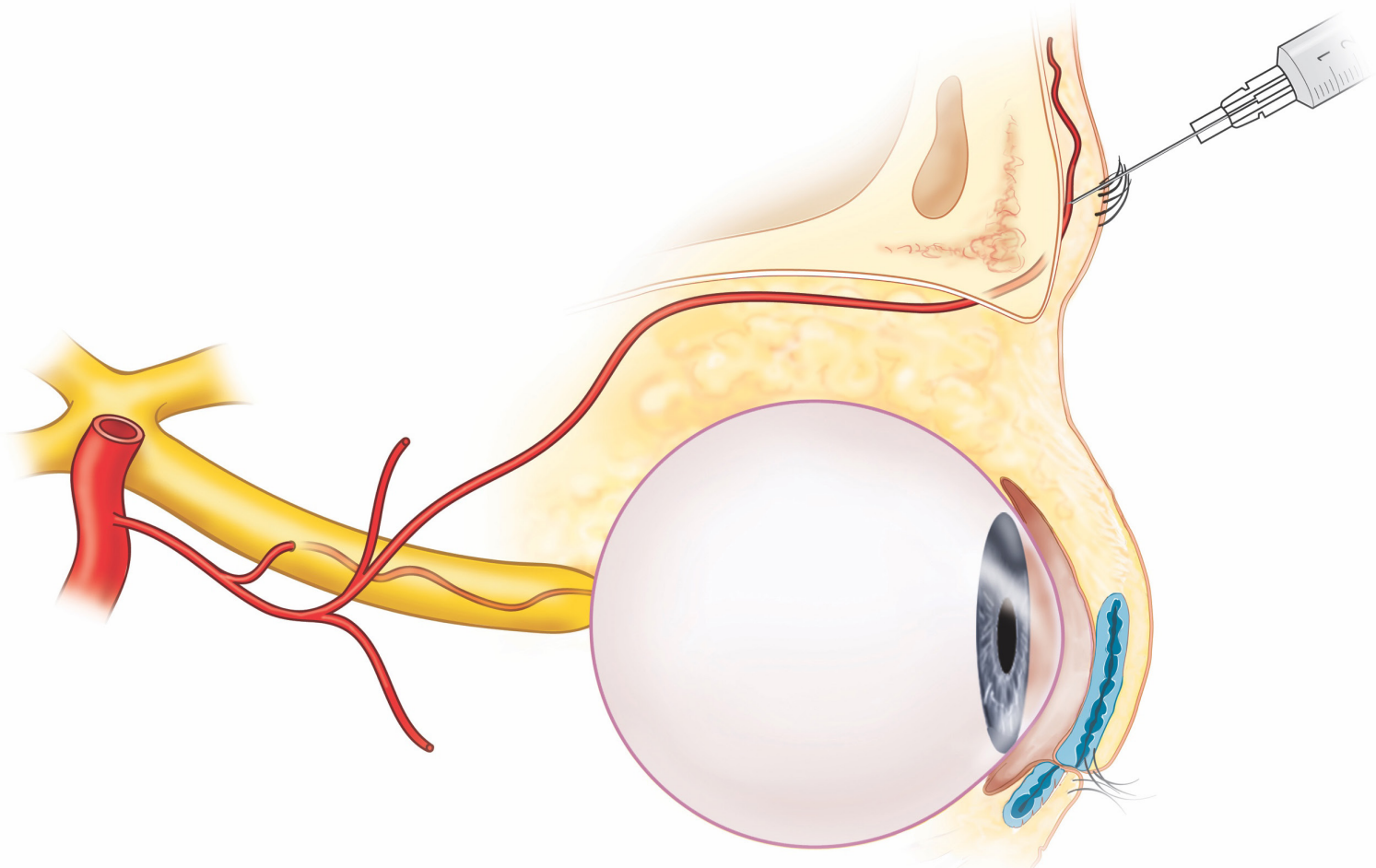

Figure 20. Mechanism of filler-induced blindness. An example of how retrograde arterial flow after injection into an ophthalmic artery branch can cause blindness. This phenomenon can also occur with injection of other periorbital vessels

occlusion resulting in either soft tissue loss or blindness is a rare but known complication of filler injection. This devastating outcome is minimized by safe injection techniques (aspiration prior to injection, avoidance of anatomic areas that risk vascular compromise via compression or embolization, and avoidance of injecting large volumes). However, if this complication does occur, early detection is key with immediate hyaluronidase injection if a hyaluronic acid filler was used at the first sign of color or sensory change or onset of pain. The treatment algorithm involves 500 units per anatomic unit every hour until resolution of soft tissue changes are noted. Blindness is the most feared complication of fillers and occurs due to retrograde arterial flow after injection into an ophthalmic artery branch [Figure 20]. If this should occur, there is no known consistent means of reversing filler induced visual changes. Retrobulbar hyaluronidase has been suggested, but it is a controversial means of treatment, and its use in unpracticed hands is illadvised. Yet, immediate ophthalmologic evaluation to reduce intraocular pressure and encourage return of blood flow is recommended ${ }^{[48]}$. A photo of the senior author's in-office filler emergency kit is included [Figure 21]. Because the success of hyaluronidase injection for clearing an intravascular occlusion is not certain, prevention of embolism is critical. The careful injector should aspirate prior to injection to ensure the needle is not intraarterial, move the needle during injection to avoid injecting a large volume in one area, and apply only gentle pressure to avoid forcing filler into a false, undesired plane.

\section{Fat grafting}

Fat grafting is another tool for facial rejuvenation and volume restoration. It has the advantages of availability and cost effectiveness, but is limited by unpredictable ultimate retention. Many of the injection techniques are similar to those used for fillers. However, special consideration must be given to the harvesting and processing techniques that optimize fat graft retention and to the methods of minimizing complications specific to fat grafting. 


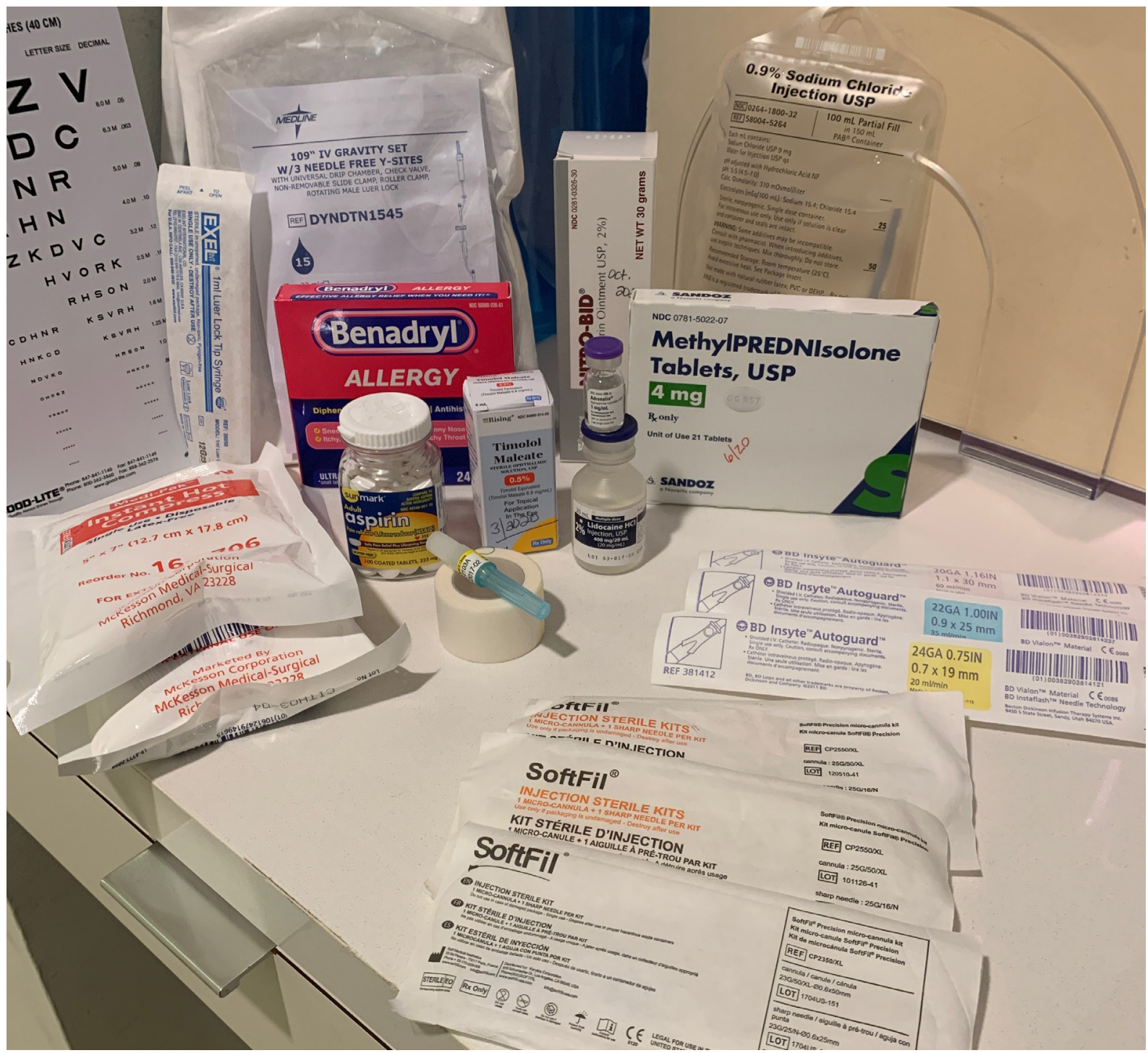

Figure 21. Emergency Vascular Occlusion/Ischemic Event Kit. Having both a protocol and Emergency Vascular Occlusion Kit (above) is a necessity in any practice utilizing injectable fillers. Staff should be familiarized with the protocol(s) and location of emergency supplies. Listed are the contents, at a minimum, included in the senior author's vascular occlusion emergency kit: hot pack(s), nitroglycerin paste. aspirin (325 mg), medrol dose pack, hyaluronidase (750 units), and a 25-gauge two-inch microcannula

When harvesting fat for grafting, a careful technique should be used to minimize adipocyte trauma and maximize viability. Although harvest location has not been shown to impact viability, ultrasound- or power-assisted liposuction should be avoided due to the trauma inflicted upon adipocytes. Fat is then prepared by gravity separation, centrifugation or Telfa rolling - none of which have been shown to more or less effective for increasing ultimate graft retention as long as centrifugation is limited to less than $3000 \mathrm{rpm}$ and less than three minutes. Fat should be injected using 18-20 gauge blunt-tipped cannulas and in small $0.1 \mathrm{~mL}$ aliquots to reduce the risk of fat necrosis or nodule formation ${ }^{[51]}$. In the periorbital region, injection should be performed deep to the orbicularis and overcorrection should be avoided. Suborbicularis injections are critical as injection just deep to the skin will result in visible contour abnormalities and nodule formation ${ }^{[52]}$.

The most common complication of fat grafting, particularly periorbital fat grafting, is contour abnormality that is visible or palpable. Liposuction is rarely a successful management strategy. Triamcinolone injections 
can be attempted but direct excision may be necessary. Vascular compromise can occur, similar to synthetic fillers, but is more difficult to treat as no enzymatic digestion is available ${ }^{[52]}$. Importantly, patients should be counseled that multiple treatments may be needed to achieve the desired effect.

\section{Reduction}

\section{Deoxycholic acid}

When performing facial rejuvenation, volume reduction is most commonly indicated in the submental region. Deoxycholic acid $\left(\right.$ Kybella $^{\circ}$ ) is an injectable agent that disrupts adipocyte cell membranes and is used for noninvasive fat reduction.

Deoxycholic acid injections are indicated for the pre-platysmal fat, but should not be injected deep to the

platysma. They can be performed in men or women and do not require anesthesia. The ideal patient has good skin elasticity in order to expect skin retraction following the procedure ${ }^{[53]}$.

When injected into the submental fat, an average of three to four rounds of injections are needed with decreasing amounts of product and a reduction of $92.8 \%$ of submental fat volume is achieved ${ }^{[53]}$. Injections are performed in a pre-marked grid with approximately two milligrams of product per square centimeter, amounting to up to fifty injection sites.

Risks of the procedure include swelling, which occurs within $48 \mathrm{~h}$ of the procedure, lasts an average of 2 weeks, and results in an average $8.68 \%$ volume increase. In spite of this post-procedure swelling, patient satisfaction was significantly improved with deoxycholic acid injections. Other reported risks include nerve paralysis, dysphagia, dysphonia, and wound formation, but these are rare and reversible ${ }^{[53]}$.

\section{Cryolipolysis}

Submental fat reduction can also be achieved through cryolipolysis. Cryolipolysis (CoolSculpting ${ }^{\circ}$ ) works by controlled cooling to selectively damage adipocytes while sparing surrounding tissue.

Prior to treatment, the area is cleaned and a gel is applied. The procedure is then performed using a small applicator intended for the submental region and lasts approximately forty-five minutes. Massage is required following treatment to rewarm and reshape the tissue which will be significantly swollen immediately upon probe removal ${ }^{[54]}$.

Studies of submental cryolipolysis demonstrated a volume reduction of $20.3 \%$ that was stable by 6 weeks following treatment. Seventy-six percent of patients were satisfied with the results of their treatment ${ }^{[54]}$. Patient selection is critical in this regard as patients with significant overlying skin laxity will have worsened laxity following fat dissolution.

Adverse events following cryolipolysis most commonly include erythema, edema, and paresthesias. All of these are self-limiting. There have been cases of paradoxical fat hypertrophy following treatment, but these cases are rare, affecting approximately $1 \%$ of patients. Risk factors for this complication include male gender, history of cryolipolysis, and Hispanic origin. Other risk factors, such as abdominal region and large handpiece, do not apply to the submental region ${ }^{[55]}$.

\section{OTHER TECHNIQUES}

\section{Platelet rich plasma}

\section{Mechanism}

Platelet rich plasma (PRP) is an autogenous solution derived from a sample of a patient's own blood. It is theorized to work to promote wound healing by releasing secretory granules that contain growth factors to induce tissue regeneration and collagen formation that, altogether, increase skin thickness ${ }^{[56]}$. 


\section{Indications}

PRP has been used in facial rejuvenation to reverse skin aging and to supplement fat grafting for increased retention $^{[57]}$. There have been seven studies of PRP for rejuvenation of aging facial skin. These studies varied in their number of treatments (one treatment or three treatments every two to four weeks) and in their sites of intradermal injection. Findings included improved skin texture and elasticity, decreased rhytids, improved skin color, improved fullness, and normalization of pigmentation ${ }^{[57]}$. Given the heterogeneity of these studies, variable samples sizes, and short follow up duration, additional studies are needed.

There have also been seven studies of PRP supplementation of fat grafting. The ratio of fat to PRP in these studies varied widely from 2:1 to 10:1. Injection sites included nasolabial folds, malar region and temporal region. Most of these studies evaluated results after only one treatment. Results were variable with some reporting aesthetic improvement and others reporting increased resorption. Of note, these studies did not have a control group so results should be interpreted with caution ${ }^{[57]}$.

\section{Procedure}

If planning to offer PRP for either of these indications in one's practice, it is important to realize that standardized, evidence-based protocols have yet to be developed. Of the available articles on PRP in aesthetic surgery, the volume of blood drawn, PRP isolation procedure, and PRP yield varied widely. Therefore, each surgeon is encouraged to determine the protocol that is most efficient and effective in his or her practice ${ }^{[57]}$.

In terms of complications, patients may experience self-limiting redness, swelling, or bruising, but there were no reported serious complications secondary to PRP harvest or injection ${ }^{[57]}$.

\section{Microinjection devices}

\section{Mechanism}

Microinjection (e.g., Aquagold ${ }^{\grave{\partial}}$ ) is the combination of microneedling, which was discussed previously, and the delivery of active ingredients such as hyaluronic acid, peptides, and other active ingredients. By injuring the stratum corneum, this technique is theorized to allow for more efficient drug delivery into the deeper layers of the dermis where they can exert their intended effect.

\section{Indications}

Hyaluronic acid microinjection is intended to increase dermal thickness and skin quality. Peptide microinjection is beneficial as peptides cannot naturally permeate the skin barrier. These peptides have various applications including stimulation of collagen production. However, both these indications are theoretical and have yet to be fully studied ${ }^{[38]}$. Other skincare ingredients, such as antioxidants and retinols, have also been hypothesized to benefit from microinjection. Early studies have confirmed their effective delivery. However, the benefit of this system is yet to be confirmed ${ }^{[58]}$.

\section{Outcomes}

While this technique requires further study, the use of microneedling with drug delivery or with subsequent application of active skincare ingredients has been shown to increase the penetration of these agents and is a relatively safe procedure with risks including irritation, erythema, swelling and bruising that are self-limiting.

\section{CONCLUSION}

With the growing demand for noninvasive rejuvenation procedures, it is imperative that plastic surgeons remain experts in the safest and most effective ways in which to use these emerging technologies. This review article provides an overview of the modalities available for skin resurfacing, skin tightening, rhytid 
reduction, and volume restoration, as well as novel modalities to combat facial aging. For most patients, a combination of these interventions will deliver optimal outcomes. Our goal is to prepare plastic surgeons to continue to offer the best and safest results to patients who desire appreciable results with minimal downtime.

\section{DECLARATIONS}

\section{Authors' contributions}

Manuscript drafting: Farber SE

Manuscript outlining: Brown E, Krochonis J, McConville R

Figure compilation and manuscript revisions: Epps MT

Manuscript revisions, figures, and videos: Codner MA

\section{Availability of data and materials}

Not applicable.

\section{Financial support and sponsorship}

None.

\section{Conflicts of interest}

All authors declared that there are no conflicts of interest.

\section{Ethical approval and consent to participate}

Not applicable.

\section{Consent for Publication}

Written consent for publication was obtained for all images and videos.

\section{Copyright}

(c) The Author(s) 2020.

\section{REFERENCES}

1. American Society of Plastic Surgeons Release. 2018 Plastic surgery statistics report. Available from: https://www.plasticsurgery.org/ documents/News/Statistics/2018/plastic-surgery-statistics-full-report-2018.pdf. [Last accessed on 2 Dec 2020]

2. Sambandan DR, Ratner D. Sunscreens: an overview and update. J Am Acad Dermatol 2011;64:748-58.

3. Hughe MCB, Williams GM, Baker P, Green AC. Sunscreen and prevention of skin aging: a randomized trial. Ann Intern Med 2013;158:781-90

4. Wang SQ, Balaqula Y, Osterwalder U. Photoprotection: a review of the current and future technologies. Dermatol Ther 2010;23:31-47.

5. Xu S, Kwa M, Agarwal A. Sunscreen product performance and other determinants of consumer preferences. JAMA Dermatol 2016;152:920-7.

6. Mancuso JB, Maruthi R, Wang SQ, Lim HW. Sunscreens: an update. Am J Clin Dermatol 2017;18:643-50.

7. Verschoore M, Nielson M. The rationale of anti-aging cosmetic ingredients. J Drugs Dermatol 2017;16:94-7.

8. Nusgens BV, Humbert P, Rougier A, et al. Topically applied vitamin C enhances the mRNA level of collagens I and II, their processing enzymes and tissue inhibitor matrix metalloproteinase 1 in the human dermis. $J$ Invest Dermatol 2001;116:853-9.

9. Murray JC, Burch JA, Steilen RD, et al. A topical antioxidant solution containing vitamins C and E stabilized by ferulic acid provides protection for human skin against damage caused by ultraviolet radiation. J Am Acad Dermatol 2008;59:418-25.

10. Hubbard BA, Unger JG, Rohrich RJ. Reversal of skin aging with topical retinoids. Plast Reconstr Surg J 2014;133:481-90e.

11. Chiu A, Kimball AB. Topical vitamins, minerals and botanical ingredients as modulators of environmental and chronological skin damage. Br J Dermatol 2003;149:681-91.

12. Leyden J. Adapalene in clinical practice. Cutis 2001;68:7-9s.

13. Ditre CM, Griffen TD, Murphy GF, et al. Effects of alpha-hydroxy acids on photoaged skin: a pilot clinical, histologic, and ultrastructural study. J Am Acad Dermatol 1996;34:187-95.

14. Smith WP. Epidermal and dermal effects of topical lactic acid. J Am Acad Dermatol 1996;35:388-91. 
15. Graf J. Antioxidants and skin care: the essentials. Plast Reconstr Surg J 2010;125:378-83.

16. Freedman BM. Hydradermabrasion: an innovative modality for nonablative facial rejuvenation. J Cosmet Dermatol 2008;7:275-80.

17. Brody HJ. Medium-depth peeling. In: Chemical Peeling and Resurfacing. St. Louis: Mosby; 1997. pp. 109-10.

18. Hevia O, Nemeth AJ, Taylor JR. Tretinoin accelerates healing after trichloroacetic acid chemical peel. Arch Dermatol 1991;127:678-82.

19. Grimes PE. A microsponge formulation of hydroquinone $4 \%$ and retinol $0.15 \%$ in the treatment of melasma and postinflammatory hyperpigmentation. Cutis 2004;74:362-8.

20. Monheit GD. Skin preparation: an essential step before chemical peeling or laser resurfacing. Cosmet Dermatol 1996;9:13-4.

21. Hirsch RJ, Dayan SH, Shah AR. Superficial skin resurfacing. Facial Plast Surg Clinc N Am 2004;12:311-21.

22. Krochonis JB, McConville RL, Kosowski TR. Nonsurgical periorbital rejuvenation. In: Codner MA, McCord DC, editors. Eyelid and periorbital surgery. 2nd ed. New York: Thieme; 2017. pp. 483-510.

23. Pathak A, Mohan R, Rohrich RJ. Chemical peels: role of chemical peels in facial rejuvenation today. Plast Reconstr Surg $J$ 2020;145:58-66e.

24. Herbig K, Trussler AP, Khosla RK, Rohrich RJ. Combination Jessner's solution and trichloracetic acid chemical peel: technique and outcomes. Plast Reconstr Surg J 2009;124:955-64.

25. Pozner JN, DiBernardo BE. Laser resurfacing: full field and fractional. Clin Plast Surg 2016;43:515-25.

26. Preissig J, Hamilton K, Markus R. Current laser resurfacing technologies: a review that delves beneath the surface. Semin Plast Surg 2012;26:109-16.

27. Karimipour DJ, Karimipour G, Orringer JS. Microdermabrasion: an evidence-based review. Plast Reconstr Surg J 2010;125:372-7.

28. Shin JM and Kim JE. Radiofrequency in clinical dermatology. Medical Lasers 2013;2:49-57.

29. Bonjorno AR, Gomes TB, Pereira MC, et al. Radiofrequency therapy in esthetic dermatology: a review of clinical evidences. $J$ Cosmet Dermatol 2020;19:278-81.

30. Zelickson BD, Kist D, Bernstein E, et al. Histological and ultrastructural evaluation of a radiofrequency-based nonablative dermal remodeling device: a pilot study. Arch Dermatol 2004;140:204-9.

31. Beasley KL and Weiss RA. Radiofrequency in cosmetic dermatology. Dermatol Clin 2014;32:79-90.

32. Dendle J, Wu DC, Fabi SG, Melo D, Goldman MP. A retrospective evaluation of subsurface monopolar radiofrequency for lifting of the face, neck, and jawline. Dermatol Surg 2016;42:1261-5.

33. Gutowski KA. Microfocused ultrasound for skin tightening. Clin Plast Surg 2016;43:577-82.

34. Minkis K, Alam M. Ultrasound skin tightening. Dermatol Clin 2014;32:71-7.

35. Suh DH, Shin MK, Lee SJ, et al. Intense focused ultrasound tightening in asian skin: clinical and pathologic results. Dermatol Surg 2011;37:1-8.

36. MacGregor JL, Tanzi EL. Microfocused ultrasound for skin tightening. Semin Cutan Med Surg 2013;32:18-25.

37. Bunin LS, Carniol PJ. Cervical facial skin tightening with an infrared device. Facial Plast Surg Clin North Am 2007;15:179-84.

38. McCrudden MT, McAlister E, Courtenay AJ, et al. Microneedle applications in improving skin appearance. Exp Dermatol 2015;24:561-6.

39. Ablon G. Safety and effectiveness of an automated microneedling device in improving the signs of aging skin. J Clin Aesthet Dermatol 2018;11:29-34.

40. Alster TS, Graham PM. Microneedling: a review and practical guide. Dermatol Surg 2018;44:397-404.

41. Kim SE, Lee JH, Kwon HB, Ahn BJ, Lee AY. Greater collagen deposition with the microneedle therapy system than intense pulsed light. Dermatol Surg 2011;37:336-41.

42. Bonaparte JP, Ellis D, Quin JG, et al. A comparative assessment of three formulations of botulinum toxin type a for facial rhytides: a systematic review with meta-analyses. Plast Reconstr Surg J 2016;127:1125-40.

43. McConville RL, Krochonis JB, Popp ME, Kosowski TR. Cosmetic uses of botulinum toxin. In: Codner MA and McCord DC editors. Eyelid and Periorbital Surgery. 2nd ed. New York: Thieme; 2017. pp. 539-60.

44. Small R. Botulinum toxin injection for facial wrinkles. Am Fam Physician 2014;90:168-75.

45. Lamilla GC, Ingallina FM, Poulain B, Trevidic P. Anatomy and botulinum toxins injections. 2nd ed. Paris, FR: E2e Medical Publishing; 2010.

46. Jabbour S, Awaida CJ, Elkhoury JS, et al. The impact of upper face botulinum toxin injections on eyebrow height and forehead lines: randomized controlled trial and an algorithmic approach to forehead injection. Plast Reconstr Surg J 2018;142:1212-7.

47. Trevidic P, Sykes J, Criollo-Lamilla G. Anatomy of the lower face and botulinum toxin injections. Plast Reconstr Surg J 2015;136:84-91s.

48. Krochonis JB, McConville RL, Kosowski TR. Injectable fillers. In: Codner MA, McCord DC editors. Eyelid and Periorbital Surgery. 2nd ed. New York: Thieme; 2017. pp. 511-38.

49. De Maio M, DeBoulle K, Braz A, Rohrich RJ. Facial assessment and injection gudie for botulinum toxin and injectable hyaluronic acid fillers: focus on the midface. Plast Reconstr Surg J 2017;140:540-51e.

50. De Maio M, Wu WTL, Goodman GJ, Monheit G. Facial assessment and injection gudie for botulinum toxin and injectable hyaluronic acid fillers: focus on the lower face. Plast Reconstr Surg J 2017;140:393-405e.

51. Donofrio LM. Techniques in facial fat grafting. Aesthet Surg J 2008;28:681-7.

52. DiFrancesco LM. Fat grafting and complications in the periorbital region. In: Codner MA, McCord DC, editors. Eyelid and Periorbital Surgery. 2nd ed. New York: Thieme; 2017. pp. 561-86.

53. Grow JN, Holding J, Korentager R. Assessing the efficacy of deoxycholic acid for the treatment of submental fat: a three-dimensional study. Aesthet Surg J 2019;39:1400-11.

54. Jain M, Savage NE, Spiteri K, Snell BJ. A 3-dimensinoal quantitative analysis of volume loss following submental cryolipolysis. Aesthet 
Surg $J$ 2020;40:123-32.

55. Stroumza N, Gauthier N, Senet P, et al. Paradoxical adipose hypertrophy (PAH) after cryolipolysis. Aesthet Surg J 2018;38:411-7.

56. Puri N. Platelet rich plasma in dermatology and aesthetic medicine. Our Dermatol 2015;6:207-11.

57. Motosko C, Khouri K, Poudrier G, Sinno S, Hazen A. Evaluating platelet-rich therapy for facial aesthetics and alopecia: a critical review of the literature. Plast Reconstr Surg J 2018;141:1115-23.

58. Kim M, Yang H, Kim H, et al. Novel cosmetic patches for wrinkle improvement: retinul retinoate- and ascorbic acid-loaded dissolving microneedles. Int J Cosmet Sci 2014;36:207-12. 